\title{
Single-cell sequencing of the adult killifish (N. furzeri) brain identifies an atypical progenitor, glial and neuronal heterogeneity
}

\author{
Rajagopal Ayana $^{1,2}$, Jolien Van houcke ${ }^{1}$, Caroline Zandecki ${ }^{1,2}$, Valerie Mariën ${ }^{1}$, Eve Seuntjens ${ }^{2,3^{*}}$, \\ Lutgarde Arckens ${ }^{1,3^{*}}$
}

${ }^{1}$ Laboratory of Neuroplasticity and Neuroproteomics, Animal Physiology and Neurobiology Section, Department of Biology, KU Leuven, Belgium

${ }^{2}$ Laboratory of Developmental Neurobiology, Animal Physiology and Neurobiology Section, Department of Biology, KU Leuven, Belgium

${ }^{3}$ Leuven Brain Institute, Leuven, Belgium

Email: rajagopal.ayana@kuleuven.be

*Corresponding authors: Lutgarde Arckens (lut.arckens@kuleuven.be), Eve Seuntjens (eve.seuntjens@kuleuven.be)

\section{Highlights}

- Single-cell transcriptomics reveals four radial glial and one non-glial progenitor cell types relevant to the neurogenic potential of the adult killifish telencephalon

- Iterative clustering of neuronal cell types identified two immature and multiple mature neuron subclasses

- Lineage inference analysis of progenitor cell types suggests a neuroepithelial-like radial glia population as the start point of neuro- and gliogenesis, that generates the proliferative nonglial progenitor

- Killifish-specific marker genes are identified for all the telencephalic cell types, with a special emphasis on markers for the non-glial progenitor, neuron subclasses and radial glia sub-types

\begin{abstract}
The African turquoise killifish uniquely combines a short lifespan with vertebrate-specific features, including age-dependent loss of neuroregenerative capacity, that are missing from the currently used model organisms. In this study, we investigate the cellular landscape that shapes adult neuro- and gliogenesis using single-cell sequencing. Our analysis identifies seventeen cell types including neuronal cells (NC), and progenitors (PC) of glial and non-glial nature in the adult killifish telencephalon. PC subclustering unveils four radial glia types, one atypical non-glial progenitor (NGP) and two clusters representing transitioning states. NC subclustering classified neurons into immature and mature excitatory or inhibitory sub-clusters. Using lineage inference analysis, we discovered neuroepitheliallike radial glia to be the source of neuro- and gliogenesis, and a central role for NGP. Our findings are evidence for specialized progenitors within telencephalon and the data is accessible via an online database, providing a resource to understand normal brain function, as well as the role of cellular relationships in response to injury and disease.
\end{abstract}

\section{Keywords}

Single-cell sequencing; killifish; Nothobranchius furzeri; neurogenesis; non-glial progenitors; telencephalon; cell markers 


\section{Introduction}

Adult neurogenesis is a complex biological process involving the integration of new neurons to the existing neural network. It is established that postnatal neurogenesis in higher order organisms including fish is focused to specific niches in the brain, and the extent of the process varies from species to species ${ }^{1,2}$. In mammals, neurogenesis is largely confined to two neurogenic zones namely, the subgranular zone (SGZ) of the hippocampal dentate gyrus and the ventricular-subventricular zone (V-SVZ) of the lateral ventricles ${ }^{3,4}$ albeit non-canonical sites do exist ${ }^{5}$. In the dentate gyrus, new neurons integrate in a controlled manner and are functionally relevant for memory and cognition. Many studies have implicated dysfunction of adult hippocampal neurogenesis in an increasing number of brain disorders, such as major depression, and neurodegenerative diseases ${ }^{6}$. Thus, stem cell and neuroregenerative therapy can be utilized to improve the condition of patients ${ }^{7}$. The regenerative capacity in the adult mammalian brain is however limited. It is therefore extremely important to elucidate which regenerative strategies are used in vertebrate regeneration-competent animals, like urodeles and fish. Especially teleost fish have gained much attention in regenerative medicine since $70 \%$ of coding genes of zebrafish are conserved ${ }^{8}$, whilst killifish share $60 \%$ of genes with humans ${ }^{9}$. Similar to mammals, the teleost telencephalon is divided into a dorsal pallium and a ventral subpallium. The dorso-lateral pallium is thought to be the equivalent of the mammalian SGZ, while the subpallium is homologous to the mammalian SVZ and hence, the telencephalon is a favorable model of study ${ }^{10,11}$.

Brain topology and cell types are conserved between mammals and teleost fish. However, the telencephalon in teleosts develops by eversion or outward folding so that the ventricle surrounds the brain parenchyma and the proliferative ventricular zones (VZ) line the border of each hemisphere ${ }^{12}$. In zebrafish, distinct proliferative zones differ in the rate of development of new cells ${ }^{12}$, and the apical radial glia (RG) form the primary progenitor population ${ }^{13}$. Particularly, RG whose cell bodies are located close to the ventricle, have long processes that extend towards the pial surface of the telencephalon, where they terminate on the walls of blood vessels or at the pial surface ${ }^{14}$. RG occasionally divide and are the equivalent of adult neural stem cells (NSCs) in mouse neurogenic zones. However, these NSCs that are $\mathrm{GFAP}^{+}$are mostly quiescent and give rise to a proliferative active NSC or a transiently amplifying progenitor type by asymmetric division ${ }^{15}$. Neuroepithelial cells, neural progenitor cells inherited from embryogenesis, help in constant supply of new RG in the VZ that later enter into quiescence and serve as neurogenic NSCs ${ }^{14}$. Interestingly, a recent study on the African turquoise killifish (Nothobranchius furzeri) discovered that it is not RG but another highly proliferative population termed non-glial progenitors (NGP) that actively drives the neuro-differentiation process in the dorsal telencephalon (pallium) ${ }^{16}$. These cells, observed in juvenile killifish at the apical surface in between RGs, are characterized by a more immature morphology and express several markers of division, including PCNA, and neural progenitor markers SOX2, MCM2, MKI67, while being devoid of pan-astroglia/RG markers like GLUL, FABP7 or GFAP ${ }^{16}$. Theoretically, NGP could be regarded similar to mammalian intermediate progenitor cells (IPC) which are known for amplifying the stem cell progeny before differentiating into neurons or glia. However, they seem to be full of self-renewal and neurogenic potential, resembling early neuroepithelial progenitors. Additionally, they have an apical domain, contacting the ventricle and express NESTIN, thus, possibly representative of neuro-epitheliallike cells rather than IPCs ${ }^{16}$. Both options hold merit but remain unexplored in adult killifish. Since the turquoise killifish is fast growing, reaching its full body size ( 2.5 inches) in about 6 weeks ${ }^{17}$, it is of utmost importance to discover which progenitors types support such rapid neurogenesis and attain neuroregenerative ability at young adult age, yet lose this at old age ${ }^{9}$. This kind of CNS regenerative ability typically exhibited by teleosts is potentially of high clinical value, and cannot be easily studied using mammalian models ${ }^{18}$. Therefore, investigating the cellular heterogeneity of the adult brain will be crucial to assess how different cells would change with age or disease, which molecular players will interplay to establish neuroplasticity and whether these can later be adaptable to neurotherapeutics.

In this study, we utilized single-cell sequencing to understand the multicellular complexity of the adult killifish telencephalon (Figure 1A). Using this high-throughput technology, we could establish an array of cellular markers for known and novel killifish brain cell types including NGP, distinctive glial subtypes, mature neurons and newborn neurons. To further refine the clustering, we performed iterative sub-clustering followed by lineage inference analyses of progenitor-like (PCs) and neuronal 
(NCs) cells separately and found 4 RG subtypes and novel cell types marking the transition between PCs and neurons. Our results elaborate previous research conducted on both juvenile and adult killifish discussing the expression of primary progenitors and glial markers in NGP and RG respectively ${ }^{9,16}$, and decode the relationship between these two progenitor classes. Extensive datasets like ours can be used for detailed investigations of the young adult killifish brain as well as act as an updated database for further research in the direction of neuroregenerative medicine. Finally, understanding the active cell mediators of this fast-growing (young) brain will prove vital to understand normal brain function and for proposing novel neurotherapeutics.

\section{Results}

\section{Sc-sequencing reveals mammalian-like cell types in the adult killifish telencephalon}

To obtain unbiased knowledge about the different cell types in the adult killifish brain, we performed single-cell RNA sequencing using the 10X Chromium method (Figure 1A). In our efforts, we optimized the single-cell dissociation protocol and obtained high viability in telencephalon cell suspensions (> 97\%) of 6-week old killifish. After sequencing, the sequenced reads were mapped to the killifish reference genome (Nfu_20140502). Mapping statistics showed that only 45\% of the single-cell reads confidently mapped to the available $N$. furzeri transcriptome (available through reference gene annotations). In order to improve the read mapping, we additionally performed Single Molecule, RealTime (SMRT) Sequencing and Iso-Seq analysis that renders full-length cDNA sequences (no assembly required) to characterize full length transcript and isoforms across an entire transcriptome of the 6-week adult telencephalon. As a result, we attained better genome and transcriptome coverage and increased the single-cell read mapping from 45 to $69 \%$ (Suppl. table 1). We recovered a total of 2928 single cells with an average of 33,000 mean reads per cell and 531 genes per cell (Suppl. table 1).

Cell QC and Clustering analysis was performed using Seurat. After removing cells with minimum and maximum thresholds for nUMI, nGene, ribosomal and mitochondrial RNA genes, we obtained 2923 high-quality cells (Suppl figure 1A, 1B, 1C). We further performed scaling and normalization, and clustered the cells. TSNE clustering revealed 17 clusters (resolution $=0.6$ ) using Principal Components $=20$, and found $59 \%$ of all cells to be of neuronal type (Figure 1B, C1, C2, Suppl. Figure 1D). Clustering identified that the different neuronal clusters (Neuronal1-5) had transcriptionally similar profiles, suggesting these cell types are more related (Suppl figure 1E). On the contrary, the gene profiles of the glia clusters (Glial1-4) and the highly proliferative cluster named NGP were found to be more divergent (Suppl. figure 1E, Suppl. table 2). The top 20 differentially expressed genes per cell cluster marked each population distinctly (Suppl. figure 1F). Notably, the top marker genes in NC clusters were downregulated in progenitors and vice versa suggesting that TSNE-based cell separation was successful (Figure 1D, Figure 2A, Suppl. figure 2A). These marker genes and particularly all genes under the criteria (adj. p-value $\leq 0.05, \log _{2} \mathrm{FC} \geq 1.2, \leq-1.2$ ) were used in identification of these cell types.

We found 5 neuronal clusters $\left[\mathrm{MAP}^{+}, \mathrm{SYPA}^{+}\right], 4$ radial glia clusters $\left[\mathrm{GLUL}^{+}, \mathrm{CX}^{+} 3^{+}\right.$or $\mathrm{FABP}^{+}{ }^{+}$] and one hyper-proliferative cluster $\left[\mathrm{PCNA}^{+}, \mathrm{HMGB}^{+} \mathrm{A}^{+}\right]$. In addition, we also found a separate glial $\left[\mathrm{GLUL}^{+}, \mathrm{VIM}^{+}\right.$] cluster with a noticeable gene signature comprising opsin genes $\left[\mathrm{RGRA}^{+}, \mathrm{RRH}^{+}\right]$, immediate early genes (IEGs ${ }^{+}$, including fos, jun) and heat-shock proteins [HSPs ${ }^{+}{ }^{19}$. This cluster, was named retinal-like radial glia (RR) and contained gene changes associated to single-cell tissue dissociation protocol, thus, was not considered for further downstream analysis ${ }^{19}$. Another cell population expressed glial as well as neuronal markers (not annotated, NA=64). We also identified microglia (MG) $\left[\mathrm{APOEB}^{+}, \mathrm{AIF} 1 / \mathrm{IBA}^{+}, \mathrm{LCP}^{+}\right]$and oligodendrocyte precursor cell (OPC/OD) $\left[\mathrm{OLIG} 1 / 2^{+}, \mathrm{CD}^{+} \mathrm{B}^{+}, \mathrm{SOX}_{10}{ }^{+}\right]$clusters (Figure 1D, Suppl. table 2, Suppl. figure 2B, 2C). We also found 3 types of vasculature-related cells which were named Vasculature1-3 (Figure 1D, Suppl. figure 2D). Since the killifish is a fairly new animal model, we provide marker genes specific to the different cell types present in the telencephalon (Figure 1D, Suppl. table 2). In this way, our initial clustering revealed several neuronal, glial and vascular cell types, and also reflects a cellular similarity between this unique teleost and mammals.

\section{Diverse expression profiles of four RG sub-types identified in the killifish telencephalon}

In order to obtain a more detailed view on progenitor cell diversity, we merged and sub-clustered all potential progenitor-like clusters (PCs: Glia 1/2/3/4, NGP, $n=559$ cells) that were expressing RG or 
proliferation markers (Figure 2A, 1D). Iterative TSNE clustering clearly separated cell clusters into 7 subtypes at an optimized resolution 0.8 (Figure 2B), and revealed the presence of 4 radial glia-like cell types. Overall, we found that these RG subtypes variably expressed FABP7A (BLBP), VIM, SLC1A2 (GLT1), CX43 (TJP1), S100B, GLUL (GS) (Figure 2C) which are known teleost RG and mammalian astrocyte marker genes ${ }^{20}$. We will now describe the distinctive expression profiles of the 4 radial glialike sub-clusters which were found, annotated as Astro-RG1, Astro-RG2, NE-RG3 and EPD-RG4. Markers such as SLC1A2, CX43 and GLUL are known to label mature astroglia in mammals, and also radial glia in juvenile killifish ${ }^{16}$. Astro-RG1 and Astro-RG2 clusters were found to contain these markers at a high expression making both RG clusters candidate astroglia in killifish (Figure 2C). Specifically, the candidate astroglia shared expression of CX43, with higher expression in Astro-RG1 which is also the larger astroglia cluster in the whole dataset (Figure 2D). The density of cells expressing CX43 at high levels was comparable between these clusters (Figure 2E). Astro-RG2 distinguished themselves by separate astroglia-like markers such as SCARA3, a known mammalian cortical astrocyte marker and S100A10, which is a typical reactive astrocyte marker ${ }^{20,21}$ (Figure 2C).

We also found ependymoglia-like cells (EPD-RG4) and an RG cluster resembling neuroepithelium-like radial glia (NE-RG3). The NE-RG3 cluster contained genesets specific to neuroepithelial cells such as KRT18, SDC4, in addition to HES1 (Suppl. table 2). Additionally, radial glia self-renewal associated transcription factors WNT8B, EMX2, SIX3 and mammalian astrocyte-specific cell adhesion molecule HEPACAM were also found to be restricted to NE-RG3 ${ }^{22,23}$. EPD-RG4 was the only cluster which showed prominent expression of apolipoproteins such as APOA1, APOA2, APOEB and CLU (APOJ) in addition to expressing FABP7A (Figure 2C, Suppl. table 2). Interestingly, this cluster also showed high expression of ependymal cell specific genes such as EPD thus suggesting an ependymal glial celllike phenotype. We propose that EPD-RG4 resembles non-ciliated ependymoglia cells in the adult telecephalon as it does not express any ciliary filament-associated genes. Astro-RG1 and NE-RG3 exhibited variable expression of VIM and S100B, both higher expressed in Astro-RG1 (Figure 2A, 2E, Suppl. table 3). In addition, Astro-RG2 and EPD-RG4 had expression of FABP7A in common, with EPD-RG4 being a small (6\%) population (Figure 2D).

In killifish, RG [PCNA ${ }^{-}$have proven to be less proliferative than NGPs by 2 days post-hatching (dph) ${ }^{16}$, and we also found this to be true in case of the adult brain (Suppl. figure 3A). We further searched for overlaps with gene marker lists for quiescent RG and proliferative RG studied in zebrafish ${ }^{24}$. This confirmed that the majority of the radial glia detected in our data were non-dividing [PCNA $/ \mathrm{MKI}^{-} 7^{-}$] (Figure 2C, Suppl. table 3). Overall, we found that only a negligible portion of RG cells expressed common proliferation markers. In contrast, classical RG markers such as FABP7A, CX43, GLUL and S100B, were enriched in these potentially quiescent or postmitotic (CCND1//PCNA) RGs (Suppl. table $3)$.

\section{Non-glial progenitors account for bulk of proliferation and neurogenesis in the telencephalon}

Iterative clustering of PCs decoded NGP as the most proliferative cluster $\left(\mathrm{PCNA}^{+}\right)$, and indicates its presumptive central role between PCs and NCs within the killifish telencephalon (Figure $1 \mathrm{C} 2$ ). It was also notable that NGPs are proliferative intermediate cells with reduced SOX2 and high SOX3 expression (Suppl. figure 3A). This cluster expresses various early cell cycle genes such as CDK1, MCM2 as well as NGP cluster-specific expression of proliferation associated genes PCNA and PCNAassociated factor, KIAA0101 (Figure 2F, Suppl. table 3).

Apart from the expected enrichment of proliferation genes, neuronal fate determinant genes, such as ASCL1 and SOX4, and marker genes of the new-born neurons, such as TMSB and HMGB2B, were significantly enriched only in NGP (Suppl. Figure 3B). Interestingly, the density of NGP cells highly expressing HMGB2A, was found to be higher than that for other specific markers such as PCNA, KIAA0101 (Figure 2F). HMGB2 is strongly associated with activation of adult NSCs and NPCs in mice ${ }^{25}$, and loss of HMGB2 compromises gliogenesis and promotes neurogenesis ${ }^{26}$. Other transcription factors associated with stemness, for instance SOX2/SOX3, were found to be variably expressed in different RGs with highest expression of SOX2 in NE-RG3 and SOX3 in Astro-RG1 (Suppl. Fig. 3A) suggestive of essential functions of these genes in RGs. It is interesting to note that the SOX2 ${ }^{+} \mathrm{NE}-\mathrm{RG} 3$ population is possibly quiescent due to downregulation of proliferation markers such as PCNA and high expression of aforementioned radial glia markers. Neither Astro-RG2 nor EPD-RG4 expressed 
SOX2/SOX3 indicating that other PC subtypes (NGP, Astro-RG1, NE-RG3, Inter-RG, Inter-NGP) are likely to be the primary active progenitor types of the killifish pallium.

\begin{abstract}
Notch-signaling activity in PC sub-types
In killifish larval stages, NGP express the pro-neural gene NEUROG1 and rely on NOTCH1 signaling for their maintenance, which suggests these cells have characteristics of embryonic neuroepithelial progenitors ${ }^{16}$. In order to assess the range of Notch-signaling activity among the PC-based sub-clusters, we analyzed the expression profiles of main markers of NGP, Notch1 pathway-related receptors and Notch signaling effector genes (Suppl. figure 3C, 3D). We found that in NGPs, NEUROG1 expression was specific, although not found in all cells, and NOTCH1B receptor expression was detected but, in comparison to NEUROG1, lower in expression. NOTCH1 ligands DLA, DLD were also detected, though sparsely expressed, in NGP (Suppl. figure 3C).

Mammalian basic helix-loop-helix (bHLH or Hes) activator and repressor genes, which are effectors of Notch signalling actively regulate maintenance of stem cells and promote astrocyte formation ${ }^{27}$. Therefore, we also performed specific profiling of Hes genes within the PC sub-clusters. The results revealed HES1 to be specific and highly expressed in NE-RG3, which is also a typical neuroepithelial cell trait (Suppl. figure 3D). HES5 which is mainly expressed in radial glia during embryogenesis was found to be expressed primarily in Inter-RG further comfirming its pro-RG fate commitment (Suppl. figure 3D). HES3 expression was missing from all clusters. Furthermore, HES effector genes such as HMGB2A, ASCL1B, HMGN1/3 were also found to be co-expressed in NGP, thus re-emphasizing the role of NGP as a crucial cell type downstream of NE-RG3 and essential for regulation of neurogenesis and cell fate determination in the telencephalon.
\end{abstract}

\title{
NGP and Intercell progenitors contain complementary transcription factors that orchestrate neurogenesis
}

PC-based subclustering uncovered that the transition between 2 important cell types, NGP and the mature mammalian astroglia-like cluster Astro-RG1, might involve more than one intermediate cell cluster, which we have referred to as intermediate 'intercell' clusters (Inter-NGP and Inter-RG), in accordance with the similarity in their respective gene profiles. The gene expression profile of InterNGP was more reminiscent of NGP precursor-like cells, while Inter-RG was closer to a radial glia cell (Figure 2C). These two transition cell states, Inter-NGP and Inter-RG clusters, were found to have a higher number of cells in comparison to other PCs (Figure 2D). Inter-RG uniquely expresses HES5 and ID4 genes which are downstream of Notch and BMP signaling respectively and promote glial differentiation, indicating a role of these pathways in promotion of astroglia formation in the killifish telencephalon (Figure 2C, Suppl. Figure 3E). Therefore, we trust the Inter-RG cluster could be a postmitotic transitioning population of radial glia (ID1/ID4 ${ }^{+} / \mathrm{SOX} 4 / \mathrm{TSMB}^{-}$), while inter-NGP (ID $/ \mathrm{MARCKS}^{+} / \mathrm{SOX}^{+}$) is more proliferative in nature with the goal to later give rise to hyper-proliferative NGPs (Figure 2C, Suppl. figure 3E). In the adult brain, the relative abundance (no. of cells) of NGP (12\%) was found to be comparable to the stem cell-like radial glia NE-RG3 (11\%) (Figure 2D). Further investigations in this direction will be vital to understand more about NGP self-renewal and stemness capacity.

\section{Spatial mapping of distinct RG sub-types reveals a more complex network}

The discovery of different types of radial glia in the telencephalon was further investigated spatially. We selected 1 specific marker from the top 5 markers of each cell type (RG1/2/3/4) to ascertain their spatial location at the ventricular surface (Figure 1D; Figure 3 TSNE plots). In situ hybridization for SLC1A2 and CX43 revealed the distribution of the cell bodies of Astro-RG1 and Astro-RG2 along the ventricle (Figure 3 A1, A2, B1, B2). Detection of CX43 also revealed fiber-like structures extending into the parenchyma, crucial evidence for their RG/astroglia-like nature (Figure $3 \mathrm{~B} 2$ ). The NE-RG3 cluster appears immunopositive for its top marker WNT8B, which is also a characteristic marker of neuroepithelium in mammals. Immunoreactive signal is detected in cell bodies lining the ventricle, sometimes also in apical fibers, and in the parenchyma, reflecting the nature of WNT8B as a secreted signalling protein $(4.1 \log 2 \mathrm{FC}$ ) (Figure $3 \mathrm{C} 1, \mathrm{C} 2$ ). The TSNE plot of the EPD-RG4 subtype illustrates the typical expression of APOA1 $\left(7.0 \log _{2} \mathrm{FC}\right)$ at a very high level (Figure $\left.3 \mathrm{D} 1\right)$. APOA1 expression is clearly restricted to the outer rim of the ventricle similar to where the ependymal cells reside (Figure 3 
D2). The glial nature of these cells $\left(\mathrm{FABP}^{+} \mathrm{A}^{+}\right)$indicates that they could be ependymoglia cells, and recent studies also suggest that unlike mammalian ependymal cells, teleost fish have a more diverse repertoire of cells within the ependyma ${ }^{28}$. Together these findings about the spatial location of the each RG type indicate distinctive distribution, which along with NGPs ${ }^{16}$ confirm the mosaic heterochrony in apical neural progenitors within the killifish telencephalon. Overall, marker gene in situ expression or immunostaining and TSNE-based clustering reveal that the adult killifish telencephalon contains distinct progenitor subtypes that can be spatially defined.

\section{NE-RG3 is a likely start cell type for glial cell types in the telencephalon}

Contrary to zebrafish, NGPs were recently proposed as the neuron-generating progenitor type in the adult killifish pallium and not RGs ${ }^{16,29,30}$. Specific lineage tracing of both progenitor classes was however still missing. In this direction, we performed lineage inference analysis of the 7 PC sub-clusters using Slingshot (Figure 4A). We used NE-RG3 as the root cluster for this analysis based on its neuroepithelial cell signature and known neurogenesis process in vertebrates, making it the most likely candidate (Figure 1D, Suppl. table 3). We also repeated this analysis with Inter-NGP or NGP as root clusters, but this resulted in NE-RG3 becoming a terminal cluster which is inexplicable with its stem cell-like transcriptomic profile. So with NE-RG3 as the root cluster, we could clearly elucidate that there are 2 possible lineages, each of which pass through the NGP and inter-NGP stages to reach diverse terminal cell states. The first lineage leads to the Astro-RG1 cell cluster via NGP, and this involves two intermediate cell types, which we named inter-NGP and inter-RG (Figure 4 A1). The complete list of shared or unique genes between all 4 cell types (NGP, Astro-RG1, Inter-NGP, Inter-RG) is available (Suppl. table 3). The second lineage was found to branch out post NGP to give rise to Astro-RG2, then closely followed by EPD-RG4 (Figure 4 A2). Pseudotime-based expression values were analyzed and this also indicated a difference in expression profiles within progenitors, with marker expression of NERG3 being very different to the following cluster (NGP). The next 2 clusters have relatively similar (proliferative) profiles (NGP/Inter-NGP), followed by more glial profiles in the last two clusters (InterRG/Astro-RG1) (Figure 4 B1). Pseudotime-based expression values of top 30 genes per lineage also indicate a sharp difference in profiles through the lineage until Astro-RG2 and then somewhat similar expression profiles between Astro-RG2 and EPD-RG4 due to a small set of shared genes such as FABP7A and CLU, and finally a distinctive EPD-RG4-specific gene profile (EPD $/ \mathrm{APOA}^{+}$) (Figure 4 B2). It could be hypothesized that EPD-RG4 is derived from an Astro-RG2 precursor but, the start point for which according to the lineage inference analysis would still be NE-RG3. PC-based lineages consistently included Inter-NGP and NGP as key transition states between different RG cell types. In conclusion, lineage inference analyses of PCs proved vital to decipher the links between multiple progenitor types and suggested that most radial glial cell fate decisions are being made at the stage of the highly proliferative NGP.

\section{Iterative clustering of NC-PCs revealed intermediate cells and neuronal subtypes}

To unravel the ontogeny and relationships between neuronal clusters (NCs), we performed iterative clustering of all NCs and primary root clusters from previous analysis (NE-RG3 \& NGP), thus filtering out the rest of the cells (Vasculature1/2/3, OD/OPC, MG, RR, NA, RG1, RG2, RG4) (Suppl. figure $2 \mathrm{~A})$. TSNE clustering of NC cells revealed 11 clusters at a resolution of 0.8 with the major contributing populations being mature neurons $(\mathrm{mN}$, Figure 5A, 5B). We found 11 sub-clusters, broadly comprising immature, mature neurons, and including retained primary progenitor types. Specifically, we found 2 sub-clusters between NGP and the NCs; a new cluster named Trans-N due to transitioning neuron properties [ELAVL3 ${ }^{+}, \mathrm{EOMESA}^{+}$]) and Inter-NGP. Trans-N is a large cluster (18\% cells of total Figure 5B) with low expression of pan-neuronal markers such as MAP2 and SYP, and enriched expression of immature neuron markers such as ELAVL3, TUBB5 and MIBP2. We also found other cell types such as newborn neurons ( $\left.\mathrm{NBN}\left[\mathrm{DCX}^{+}, \mathrm{EOMESA}^{+}\right]\right)$, an inhibitory neuron-like $\mathrm{mN}$ cluster (Ih-mN6 $\left.\left[\mathrm{GAD}^{+} \mathrm{B}^{+}, \mathrm{GAD}^{+}\right]\right)$and 5 other mature excitatory neuronal clusters (Ex$\mathrm{mN} 1 / \mathrm{mN} 2 / \mathrm{mN} 3 / \mathrm{mN} 4 / \mathrm{mN} 5\left[\mathrm{SYP}^{+}, \mathrm{SLC}_{\left.17 A 6 \mathrm{~B}^{+}\right]}\right.$) (Figure 5A, 5C, 5D).

Interestingly, 4 clusters (Trans-N, NBN, NGP and Inter-NGP) shed more light on neurogenic commitment or a branchpoint with specific expression of MEX3A, a crucial gene that is required for neuroblast proliferation during adult neurogenesis ${ }^{31,32}$. MEX3A was highly expressed in Trans-N/NBN and lower in NGP/Inter-NGP (Figure 5C). In the Trans-N cluster, the reduced expression of mature 
neuronal markers combined with expression of neurogenesis regulators is indicative of its crucial role in neuronal differentiation. As such, the Trans-N cluster might act as a precursor cell type to the newly born neuron cluster named NBN $\left(\mathrm{NEUROD} 2^{+}, \mathrm{TUBB}^{+}\right)$. Of note, these genes are characteristic of new-born neurons formed in the teleost pallium during neural development and, in zebrafish, they are a derivative of the radial glia mother cell ${ }^{24}$. This killifish NBN cluster also showed striking resemblance (marker expression) to zebrafish neuroblasts $\left[\mathrm{ELAVL}^{+}, \mathrm{EOMESA}^{+}, \mathrm{MIBP}^{+}\right]$as described by Cosacak et al. (Figure 5C) ${ }^{33}$.

In relation to EOMESA, it is also reported that EOMESA ${ }^{+}$basal intermediate progenitors in zebrafish $\mathrm{VZ}^{34}$ are different from the apical NGPs that are EOMESA- in killifish. We found that the new-born neurons express EOMESA, and this seems to be killifish-specific. The Trans-N and NBN clusters together exhibited features of new-born neurons transcriptionally similar to neuroblasts in postnatal neurogenesis and intermediate progenitors $\left[\mathrm{DCX}^{+} / \mathrm{NEUROD}^{+} / \mathrm{EOMESA}^{+}\right]$respectively (Figure $5 \mathrm{D}$ ). The killifish has a different IPC-like cluster comprising Trans-N and NBN, which are both derived from Inter-NGP and NGPs. We also found that unlike in zebrafish, these 2 clusters in killifish were not NEUROG $^{+35}$ (Suppl. table 4). In conclusion, we found that the NCs and PCs form closely linked lineages mediated by NGPs. Overall, we proved iterative sub-clustering of PC-NCs is an efficient way to decode the missing links between neurogenic commitment when starting from NE-RG3 and NGP clusters.

\section{Mature neuronal classes possess highly similar transcriptomic profiles}

In the top 10 genes expressed by each neuronal cell type, several pan-neuronal markers were found to be shared (Figure 5D, Suppl. table 4). We further ascertained the markers separating immature $\left(\mathrm{ELAVL}^{+}\right)$, mature $\left(\mathrm{MAP}^{+}\right)$and new-born neurons $\left(\mathrm{DCX}^{+}\right)$in the killifish telencephalon (Figure 5C, 5D). Our results further suggest that all identified neuron subtypes can be distinguished from one another via the expression of individual or combinations of genes belonging to specified categories (Figure 5D). Gene commonalities between all mature neuronal clusters (Ex-mN1, Ex-mN2, Ex-mN3, Ex-mN4, Ex-mN5 and Ih-mN6), included pan-neuronal markers such as MAP2, SYP and SV2A. MAP2 is known to label the perikarya, dendrites and axon, while SYP and SV2A label the synapse and synaptic vesicles. Mature neuronal clusters Ex-mN1, Ex-mN2, Ex-mN3, Ex-mN4 and Ex-mN5 hosted an array of features resembling excitatory neurons, with expression of markers associated with glutamate transport such as SLC17A6A and SLC17A6B (vGLUT2) (Figure 5D. Apart from this, these clusters also expressed neuropeptides and their respective receptors (Figure 5D).

Specifically, the Ex-mN1 cluster has the highest expression of SV2A and MAP2 among the mature neuronal clusters, while Ex-mN3 expresses the highest ELAVL4 level (Figure 5D, Suppl. table 4). Only Ex-mN4 and Ex-mN5 express SLC17A7 (vGLUT1) and GRIN1 (Figure 5 B2). Among all the clusters, PVALB expression appeared specific to Ex-mN4 (Figure 5D). The Ex-mN5 cluster specifically expressed GABA receptor genes GABBR1 and GABRB2 (Figure 5D). The cluster that possessed glutamate receptor expression (GRIA1) was the Ih-mN6 cluster, which also showed high expression of the GABA-synthesizing enzymes GAD1B (GAD65) and GAD2 (GAD67) characterizing it as the only inhibitory neuron population.

\section{NE-RG3 and NGP contribute to the start of the neuronal lineage}

In order to identify how these neuronal cell types are related to each other, we constructed a pseudotimebased ordered system using NC-PC subclusters. Unequivocally, NE-RG3 was taken as the start cluster in accordance with the previous PC lineage analysis and this identified 3 possible lineages. The first one involved starting with NE-RG3 and passing through the Trans-N and NGP precursor like cluster (Inter-NGP) to reach the excitatory or inhibitory neuronal states $(\mathrm{Ex}-\mathrm{mN} 1 / \mathrm{mN} 2 / \mathrm{mN} 3 / \mathrm{mN} 4 / \mathrm{mN} 5 / \mathrm{Ih}$ mN6) (Figure 5E). The second lineage describes the potential neuronal commitment lineage. This one starting from NE-RG3 progresses toward neuronal commitment associated cluster Trans-N via InterNGP and terminates in NBN (Figure 5E). The third lineage is a brief one which describes the formation of NGPs, starting with NE-RG3 followed by Inter-NGP, a probable NGP precursor cell (Figure 5E). Although Trans-N is also part of the lineage, the proportion of cells involved is negligible to the other 2 lineages.

We further examined the similarity in expression of top 50 differential genes per lineage using Pseudotime-based expression values (Suppl. figure 4). In the first lineage, higher differential expression 
was only noted toward the later point in the lineage when mature neuronal subclasses were formed (Suppl. figure 4A). In the second lineage, the formation of NBN is marked with a gradual expression rise in markers such as GAP43 (Suppl. figure 4B). Notably, we can also see the decline in expression of glial markers FABP7A and S100B (Suppl. figure 4B). The expression profiles of the third lineage clusters provides evidence to the possibility of a cyclic process between NE-RG3, NGP and Inter-NGP that might contribute to neuronal formation, while the terminal status of NGP sheds light on its selfrenewal capacity (Suppl. figure 4C). In this way, all three lineages showed differential contribution to neurogenesis by a common intermediate path, Inter-NGP/Trans-N clusters. These two clusters drove the path to neuronal commitment in 2 ways: towards a neuron becoming mature (excitatory or inhibitory), or forming a replenishable cluster, NBN. The terminal state of NBN presents a crucial finding as it adds fuel to the theory of a subtype of newly generated immature neurons acting as a reservoir for prevention of brain aging and disease in mammals ${ }^{36}$. We find that this could also hold true for killifish, although further investigations are needed. Additionally, Inter-NGP was found to act as a primary mediator that governs formation of NGPs from NE-RG3. In conclusion, this analysis deciphered relations between multiple neuronal types and was particularly useful for ascertaining the cell fate specification state of a neuron to be excitatory or inhibitory via novel intermediate cell types.

\section{Expression profiles of killifish vs. zebrafish telencephalic progenitors reveals species-specific features}

We briefly compared the 7 killifish PC-based subclusters to the 9 progenitor clusters [PC_0 - PC_8] reported in the zebrafish telencephalon-specific study ${ }^{33}$. We found similarity between the killifish NERG3 cluster to the zebrafish neuroepithelium PC cells (PC_6 and PC_8) (Suppl. table 5A). The killifish NGP and Inter-NGP clusters were reminiscent of neuroblasts (PC_3) and pre-neuroblasts with regards to the zebrafish expression profiles (PC_3 and PC_5) (Suppl. table 5B). Although NGP/Inter-NGP are more transcriptionally similar to neuroblasts/pre-neuroblasts, lineage inference analyses showed that they can also be seen as a strong bidirectional intermediate cell type which supports both neurogenesis and gliogenesis (Figure 4A, 5C, 5E). The zebrafish ependymal PCs (PC_7) express ciliogenesisassociated genes whereas we found non-ciliated ependymoglia in killifish (EPD-RG4) (Suppl. table 5D). On the other hand, the zebrafish ependymal layer is primarily made of radial glia (often called ependymoglia), which is different from mammals but identical to what we observe for killifish ${ }^{28}$.

In our dataset, we find 2 mature astroglia-like clusters (Astro-RG1, Astro-RG2), which were primarily absent from the zebrafish PC dataset. We performed this analysis using known marker genes related to mammalian astrocytes/astroglia (Suppl. figure 5). Mainly, the marker genes used to identify mature astroglia in killifish were either unnoticed or very low in expression in the zebrafish dataset, thus confirming their immature/progenitor phenotype (Suppl. table 5E, Suppl. figure 5). Previously, Chen et al. showed how astrocyte differentiation occurs from stem cell to post-mitotic astrocyte in vivo in spinal cord, and identified a role for FGF receptors in zebrafish astrocytes ${ }^{37}$. As a proof of concept in the killifish telencephalon, we scanned for overlaps with the gene orthologs of zebrafish-killifish FGF receptors and found all 5 orthologs: FGFR1A, FGFR1 (2 OF 2), FGFR2, FGFR3, FGFR4. Among these, Astro-RG1 exclusively expressed FGFR2 (high expression) and astrocyte-specific marker FGFR3. Specific expression of FGFR1A was found in NE-RG3 (Suppl. figure 3C). We did not find any RG cluster that expressed FGFR4. This shows how FGF receptor genes are differentially expressed between killifish RG types, suggesting an important role in astrocyte morphogenesis. In sum, we confirm that the diversity in RG subtypes appears unique to killifish and further investigations are vital to shed light on the structure, function and physiology of radial glia/astroglia in killifish.

\section{Discussion}

Cellular heterogeneity in the killifish brain is governed by differential activation of distinct molecular pathways in respective cell types, and so single-cell sequencing provides an in-depth perspective at single-cell resolution. One of the key findings of the unbiased clustering analysis was the spectacular cell diversity in this small region of the killifish brain. From our study, the adult killifish telencephalon contains at least 20 (including sub-clusters) different types of cells. We also identified deterministic marker expression for all PC clusters. We found common vertebrate cell types such as 
neurons, radial glia/astroglia, microglia, vasculature-associated cells, neuroepithelial cells, ependymoglia cells along with the special NGPs. We further established the unique features of the atypical NGP in killifish that make this population committed to neurogenesis (indicated by consistent expression of neuronal fate determinants). Particularly, NGP and NE-RG3 together show exceptional stem-cell potential to transform into a multitude of cell types. Since radial glia do not seem to support neurogenesis as much as NGP do in the killifish telencephalon ${ }^{9,16}$, they might be contributing to astrocytic functions based on gene expression profiling. Thus, the presumptive central role of NGP within the diverse repertoire of radial glia is intriguing. One of the most striking findings of this study is the diversity in RG subtypes. Why this unique teleost brain requires this level of diversity within RGs and how this is affected with aging and disease states remains a topic for future investigation.

Radial glia in teleosts such as zebrafish have been termed astroglia, and should be considered as an ancestral homolog of mammalian radial glia and astrocytes combined due to functional diversification ${ }^{28}$. Lately also in zebrafish, astroglia have been gaining attention, and only now it has been established that radial glia are not the only proliferative cell type ${ }^{38}$. Until recently it was assumed that bonafide astrocytes do no exist in teleosts and the presence of typical protoplasmic astrocytes was solely a mammalian brain characteristic. It has also been theorized that RGs perform the function of astrocytes in teleosts. Chen et al. recently reversed the theory by imaging astrocyte morphogenesis in the zebrafish spinal cord ${ }^{37}$. Their study shows astrocyte morphogenesis occurs from stem cell to postmitotic astrocyte in vivo, and identifies a role for Fgf receptors in vertebrate astrocytes which we find to be expressed in the presumptive killifish astroglia. Although not all fgf receptors are expressed to their full capacity in our single-cell data, the expression of FGFR2 and FGFR3 in Astro-RG1 is particularly enlightening as this is our candidate astroglial RG subtype (Suppl. Figure 3b). In mammals, FGF signaling has been shown to play a role in neural progenitors to switch cell fate towards astrocyte development ${ }^{39,40}$. In addition, FGFR3 has been found to be highly expressed in both human and mouse astrocytes ${ }^{41-43}$. Overall, we find that killifish and zebrafish have familiar progenitor profiles but the NGPs continue to be unique to the killifish. Furthermore, the discovery of telencephalic astroglia-like cells in our study is synchronous to first zebrafish astroglia observations in spinal cord and thus, widens avenues for comparative transcriptomics meta-analyses in this direction.

Notch signaling contributes to the elaboration of cellular diversity during the development of various tissues ${ }^{27}$. During mammalian neocortical development, HES5 transcriptional repressor, working downstream of Notch signaling, is known to regulate the timing of neurogenesis and gliogenesis ${ }^{44}$. From neuroepithelial cells to radial glial phenotype, the expression of HES5 increases, while another hes gene HES3 is downregulated. Taking this into consideration, we analysed various transcription and epigenetic factors associated with the pathway and found that NGP, Astro-RG1 and NE-RG3 clusters express multiple genes such as HES5, HES1, NOTCH3 respectively (Suppl. Fig. 3c). The differential Hes gene expression indicates spatial as well as developmental disparity between different radial glia cell types (Astro-RG1/Astro-RG2/NE-RG3) found in the killifish. Overall, this supports the theory that a variety of progenitors in the killifish telencephalon are responsible for the explosive brain growth and rapid neurogenesis.

Understanding which lineages can underlie successful neurogenesis in the adult brain remains an intense subject of investigation. Here, we used lineage inference analysis to ascertain the overall developmental scheme in the killifish brain model. The NC-based lineage analysis showed that a large proportion of cells still possess multipotency in the adult telencephalon stage, and neural commitment was governed by common intermediate cell types originating from apical progenitors (NE-RG3 and NGP). It also caught our attention that in killifish, the decision that a neuron would be excitatory or inhibitory comes sequentially after neuronal fate determination, and not anytime earlier. This is different from mammals where neuronal sub-types are generated in distinct locations during embryogenesis ${ }^{45}$. The PC-based lineage analysis suggests a multitude of progenitor types in a selfsustaining loop, some of which also showed region specificity (Figure 3). Interestingly, we could also assign specific branch points in neurodevelopment of killifish, thus confirming the point of neuronal commitment in NGPs. If there are any recursive loops in these lineage tracks and whether reprogramming between the PC cell types occurs remains a mystery. What is the function of these diverse types of radial glia/astroglia also needs to be further investigated. Although we validated the marker confirmation for known and novel radial glia types from our clustering analysis, further investigations will only confirm the accuracy of their lineage tracing. We have so far built a repertoire 
of marker genes specific to 20 cell types, which will be a valuable resource for the killifish and teleost community. Teleost fish, and specifically the short-lived killifish which harbor constitutive and efficient neurogenic niches in all brain subdivisions, form an atypical model to approach this question ${ }^{11,18}$. Further investigation of our data with concurrent experiment validation will be useful in identifying the molecular paradigms involved in cellular lineages of the adult killifish pallium. We believe understanding the adult brain provides a breakthrough discovery that opens avenues for novel therapeutics against aging-associated neurodegenerative diseases.

\section{Materials and Methods}

\section{Fish strains}

All experiments were performed on adult 6 week-old African turquoise killifish (N. furzeri), from the inbred strain GRZ-AD. One male was housed with three females in a multilinking Tecniplast ZebTec aquarium system under standardized conditions; temperature $28^{\circ} \mathrm{C}, \mathrm{pH} 7$, conductivity $600 \mu \mathrm{s}, 12 \mathrm{~h} / 12 \mathrm{~h}$ light/dark cycle, and fed twice a day with Artemia salina (Ocean Nutrition) and mosquito larvae (Chironomidae). All experiments were approved by the KU Leuven ethical committee in accordance with the European Communities Council Directive of 20 October 2010 (2010/63/EU).

\section{Anti-WNT8B immunostaining}

Fish were euthanized in 0,1\% buffered tricain (MS-222, Sigma Aldrich) and perfused with PBS and 4\% paraformaldehyde (PFA, 8.18715, Sigma-Aldrich, in PBS). Brains were carefully removed and fixed over night at $4{ }^{\circ} \mathrm{C}$ in $4 \%$ PFA. The following morning, brains were washed three times in PBS and transferred overnight to $30 \%$ sucrose in PBS at $4^{\circ} \mathrm{C}$. Next, brains were embedded in $30 \%$ sucrose, $1.25 \%$ agarose in PBS. Using a CM3050s cryostat (Leica), $10 \mu \mathrm{m}$-thick coronal sections were cut and collected on SuperFrost Plus Adhesion slides (10149870, Thermo Fisher Scientific). Sections were stored at -20 ${ }^{\circ} \mathrm{C}$ until the start of immunohistochemistry (IHC). For anti-wnt8b IHC, sections were first dried for 30 min at $37^{\circ} \mathrm{C}$. Next, sections were washed in AD, TBS $(0,1 \%$ Triton-X-100 in PBS) and heat-mediated antigen retrieval was performed in $1 \mathrm{X}$ citrate buffer $(2.1 \mathrm{~g}$ citric acid and $500 \mu \mathrm{L}$ Tween 20 in $1 \mathrm{~L}$ PBS, $\mathrm{pH}$ 6) for $15 \mathrm{~min}$ in the microwave. Hereafter, slices were cooled down for $20 \mathrm{~min}$ and washed with TBS. Blocking was performed for $1 \mathrm{~h}$ at RT using $20 \%$ normal goat serum (S26, Sigma-Aldrich) in Tris$\mathrm{NaCl}$ blocking buffer (TNB). Sections were incubated with the rabbit anti-wnt8b primary antibody (EMELCA Bioscience) diluted 1:200 at $4^{\circ} \mathrm{C}$ for 48 hours in Pierce Immunostain Enhancer (Thermo Fisher Scientific). Sections were washed three times with TBS and incubated with Goat anti-Rabbitbiotinylated, diluted 1:300 in TNB (E043201-8, Agilent Dako) for $45 \mathrm{~min}$. After washing with TBS, sections were incubated for $1 \mathrm{~h}$ with Streptavidin-Cy5 (SA1011, Thermo Fisher Scientific), diluted 1:500 in TNB. Cell nuclei were stained for 30 min with 4',6-diamidino-2-fenylindool (DAPI, 1:1000 in PBS, Thermo Fisher Scientific). After immunolabelling, sections were covered with a cover glass slide using Mowiol.

\section{In situ hybridisation}

Fish were euthanized in $0.1 \%$ buffered tricain (MS-222, Sigma Aldrich) and perfused with PBS and 4\% paraformaldehyde (PFA, 8.18715, Sigma-Aldrich, in PBS). Brains were carefully removed and fixed over night at $4{ }^{\circ} \mathrm{C}$ in $4 \%$ PFA. The following morning, brains were washed three times in PBS and transferred overnight to PBST $\left(0,1 \%\right.$ Tween-20 in PBS) at $4^{\circ} \mathrm{C}$. Next, brains were transferred to $70 \%$ Ethanol for $1 \mathrm{~h}$ and to $80 \%$ and $90 \%$ ethanol for $15 \mathrm{~min}$ each. Hereafter, brains were placed in $100 \%$ ethanol two times for $30 \mathrm{~min}$ and then transferred to 50/50\% xylol/ethanol for $15 \mathrm{~min}$. Next, brains were placed two times in $100 \%$ xylol for $30 \mathrm{~min}$ and transferred to $100 \%$ liquid paraffin $\left(60^{\circ} \mathrm{C}\right)$ for at least $1 \mathrm{~h}$. Brains were embedded in paraffin and $7 \mu \mathrm{m}$-thick sections were cut on a paraffin microtome (Thermo Fisher Scientific).

The in situ hybridisation was performed using DIG-labelled antisense probes targeting SLC1A2, CX43 and APOA1 with the use of an automated platform (Ventana Discovery, Roche). The following reagents were used during the automated procedure: RiboMap fixation, BlueMap detection kit, RiboCC, Reaction buffer and SSC (Roche). The amount of probe used was dependent on the target (150-300 ng). Afterwards, the slides are dehydrated by incubation in increasing amounts of ethanol (70\%-100\%) and 
xylol (100\%). To finish, the slides are mounted with Eukitt Quick-hardening mounting medium (Sigma).

\section{Single-cell suspension}

Three fish were euthanized in $0.1 \%$ buffered tricain (MS-222, Sigma Aldrich). Blood was removed by intra-cardiac perfusion with cold, sterilized PBS. Next, brains were extracted and immediately placed in cold DMEM/F12 (Life Tech, Invitrogen). Brains were transferred into sterilized, freshly prepared papain solution $\left(250 \mu \mathrm{L}\right.$ papain (Sigma Aldrich), $100 \mu \mathrm{L} \mathrm{1 \%}$ DNase I (DN25, Sigma Aldrich $\left.{ }^{\circledR}\right), 200$ $\mu \mathrm{L}$ L-Cysteine (12 mg/ mL, Sigma Aldrich), in $5 \mathrm{~mL}$ DMEM/F12 (Life Tech, Invitrogen)) and digested for $10 \mathrm{~min}$ at $37^{\circ} \mathrm{C}$. Hereafter brains were dissociated by gently triturating with a cut pipet tip and placed back for $10 \mathrm{~min}$ at $37^{\circ} \mathrm{C}$. This procedure was repeated until the brains were completely dissociated into single-cells. The suspension was then put through a polypropylene strainer $(35 \mu \mathrm{m}$, Falcon) and $2 \mathrm{~mL}$ of ice-cold, sterilized, freshly prepared washing solution (650 $\mu \mathrm{L} \mathrm{D-(+)-glucose} \mathrm{45 \%} \mathrm{(G8769,} \mathrm{Sigma}$ Aldrich $\left.{ }^{\circledR}\right), 500 \mu$ L HEPES (1M, Thermo Fisher), $5 \mathrm{~mL}$ FBS (Life Tech, Invitrogen) in 1X DPBS (Life Tech, Invitrogen) was added. Cells were centrifuged for $10 \mathrm{~min}$ at $500 \mathrm{~g}$ on $4^{\circ} \mathrm{C}$ and the supernatant was discarded. Next, debris was removed via the debris removal solution protocol (130-109-398, Miltenyi Biotec) to ensure a high viability of the suspension. Finally, cells were resuspended in cold sterilized $0.04 \%$ BSA in PBS. Cell viability was measured at the KU Leuven Genomic Core. A viability of $97.4 \%$ was retained after the whole procedure.

\section{Telencephalon SMRT-Sequencing}

The Iso-Seq method produces full-length transcripts using Single Molecule, Real-Time (SMRT) Sequencing, thus attaining high accuracy with better genome coverage ${ }^{46,47}$. We pooled 2-3 fishes (6 weeks old) and extracted whole RNA using the RNAEasy Micro kit (Qiagen), with blood. Final RNA quality and integrity were assayed using the DNA 12000 kit on Bioanalyzer (Agilent). Further, this was subjected to PACBIO SMRT Sequencing as recommended in Pacbio protocol for Sequel systems. The SMRT sequencing was performed on the PacBio Sequel at the Genomics Core at KU Leuven (Belgium) using the Clontech SMARTer PCR cDNA Synthesis Kit (recommended by Pacbio). Multiple parallel PCR reactions were set after the first strand synthesis employing the PrimeSTAR GXL DNA Polymerase kit. Next, cDNA of specific length was selected by splitting these PCR reaction products into two fractions, purifying one with 1x AMPure PB Beads and the second with 0.4X AMPure PB Beads, and subsequently mixing both purified products in an equimolar fashion. These libraries were constructed using the SMRTbell template prep kit 1.0. They were sequenced employing Sequel Sequencing, and Binding kits 3.0 on a Sequel I platform with SMRTCells V3.0 LR, allowing a movie collection time of $20 \mathrm{~h}$. The mRNAs were selected by the presence of poly-A tails, without 5 'cap selection, that was taken into account in the subsequent analysis. The reference genome, gene annotation, and GO terms were downloaded from the $N$. furzeri Information Network Genome Browser (NFINgb). We further used the recommended Pacbio ISOSEQ pipeline to analyse the sequencing data with in-house modifications. We further used the GFFCompare tool was used to create an updated killifish specific annotation file by merging the $N$. furzeri reference and Iso-Seq derived full length transcriptome from 6 week-old telencephalon and its specific annotations ${ }^{48}$. This helped us improve our final genomic annotation file and ensured higher mapping accuracy and coverage for performing Seurat-based single-cell sequencing analysis.

\section{Telencephalon 10X Genomics sequencing}

$10 \mathrm{X}$ Genomics is known to outperform other droplet-based sequencing methods in terms of bead quality and barcode detection efficiency. To prepare the samples for single-cell sequencing using 10X genomics, all cells isolated from telecephali were pooled from 3 killifishes. Subsequently, the singlecell suspension was carefully mixed with reverse transcription mix before loading the cells on the 10X Genomics Chromium system. Library preparation was done using 10X Genomics droplet-based sequencing with the 10X Chromium Single Cell 3' (v3 Chemistry) reagants kit. The cells were lysed within the droplet and they released polyadenylated RNA bound to the barcoded bead, which was encapsulated with the cell. Following the guidelines of the 10x Genomics user manual, the droplets were directly subjected to reverse transcription, the emulsion was broken and cDNA was purified using Silane beads. After the amplification of cDNA with 10 cycles, purification and quantification was 
performed. The 10X Genomics single-cell RNA-sequencing library preparation - involving fragmentation, dA-tailing, adapter ligation, and 12-cycle indexing PCR was performed. After quantification, the libraries were sequenced on an Illumina NovaSeq machine using a HighOutput flowcell in paired-end mode (R1: $26 \mathrm{bp} ; \mathrm{I7}: 8 \mathrm{bp}$; R2: $50 \mathrm{bp}$ ), thus generating 100 million fragments.

\section{Single-cell sequencing data analysis}

Raw data was processed with the Cell Ranger 3.0 software. First, we built the reference for CellRanger using the 'mkgtf' command (default parameters). The killifish genome (GRZ Assembly, 05/2015) as well as latest NCBI gene annotation (NFIN db) in conjugation with the in-house sequenced killifish ISOSEQ annotation files were used to specify the reference in the 'mkgtf' command. Specifically, the two gtf files from PACBIO long-reads specific to telencephalon and the NFIN db reference transcripts were merged using the GffCompare tool. This was followed by the 'count' command as part of CellRanger, the option of '- expect-cells' was set to 2500 (all other default options). The results included the feature barcode matrices in various formats usable for downstream analysis in R.

\section{Data Analysis with Seurat}

All matrices from CellRanger were read by the Read10X function using Seurat 4.0 package ${ }^{49}$. Initial quality check included removal of ribosomal genes (RPS/L genes) and filtration/removal of cells using the following criteria: a) cells having $>5 \%$ mitochondrial content, b) cells $<500$ and $>2500$ unique genes. We further removed genes/features detected in at least 2 cells (min.cells parameter), and filtered the top 2000 highly variable genes for further analysis. The remaining variable cells and genes were used for downstream analysis. Further, we scaled, normalized and checked variability of data using the single SCTranform function. Initially two Seurat SCTransformed objects, adult killifish and zebrafish scSequencing data as a reference (Cosacack et al.) were used to generate a combined object with IntegrateData function for marker gene identification studies. Post optimization, we only used killifish object to do primary analyses and further PC and NC subclustering. We performed unbiased clustering on the top 20 Principal components which has been represented in the form of dimensionality reductive T-distributed Stochastic Neighbor Embedding (TSNE) at optimized perplexity $=\mathrm{n} / 100^{50}$, and Uniform Manifold Approximation and Projection (UMAP) algorithms. PC or NC-based subclustering was performed by subsetting pertinent clusters from "all cells" object and rerunning the Seurat analysis.

\section{Cell type Identification}

Cel type identification related plots (Feature plots and dot plots) were generated by Seurat and cell types were determined by the expression of tested marker genes in the laboratory that define specific cell types and known expression in related species (zebrafish) and humans. We also removed a population of cells occurring to contamination ${ }^{19}$. The marker genes for all resultant cell populations (All cells, PCs, NCs) were calculated by using FindAllMarkers and FindMarkers function with options min.pct $=$ 0.25 , thresh.use $=0.25$ and test.use $=$ MAST. Using the FindMarkers and FindAllMarkers function, we ascertained the ranking of all differentially expressed marker genes (threshold: PCT $\geq 0.25$ ) per cell cluster, with associated significance value (corrected $\mathrm{p}$-value $\leq 0.05$ ). The same parameters were applied for the sub-clustering analyses.

\section{Lineage inference analysis with Slingshot}

To elucidate the cell lineage on pseudotime, we made a subset of clustered PC cells ( further converted that Seurat object to a Slingshot object via Slingshot ${ }^{51}$. Primarily, this is done on the basis of the differential gene expression between cell types by constructing a minimum spanning tree (MST) on cells in a reduced-dimensionality space created by independent component analysis (ICA), and orders cells via a PQ tree along the longest path through this tree. The subset of all cells included NGP, Astro-RG1/2, NE-RG3 and EPD-RG4 in case of PCs. The 'start.cluster' was set as 'NERG3/Inter-NGP/NGP' and multiple lineages in 'curves' mode were analyzed. The subset of all cells included NGP, NE-RG3, Neuronal1-5 in case of NCs. The 'start.cluster' was set as 'NE-RG3' and multiple lineages in 'curves' mode were analyzed. The estimated size factor set to 0.6 as the data were already normalized by Seurat. The cells were colored according to the colors on the Seurat TSNE plots. We also constructed a cluster tree to seek the molecular distance between each cluster using the BuildClusterTree function. 


\section{Comparisons with zebrafish progenitors dataset}

The progenitors dataset from zebrafish was scanned for gene overlaps with killifish progenitor profiles found systematically. We briefly compared our 7 PC-based clusters to 9 progenitor clusters reported for the zebrafish telencephalon [PC_0 - PC_8] by searching for the presence or absence of progenitor cell type markers as described in a recent zebrafish progenitor focused study ${ }^{33}$. Presence or absence was highlighted. Additionally, we also compared to check if killifish astroglia-like cells were present in the zebrafish dataset.

\section{Data Availability}

The 10X sequencing data have been deposited in GEO (http://www.ncbi.nlm.nih.gov/geo/) under BioProject ID PRJNA718608.

\section{Author Contributions}

R.A.: Conceptualization, experimental design, bioinformatics analysis, database development, data visualization, writing: draft, review \& editing. J.V.H.: Single-cell suspension preparation, Immunohistochemistry experiment, writing: review \& editing. C.Z.: ISH experiment, writing: review \& editing. V.M.: Review \& editing. L.A, E.S.: Study supervision, conceptualization, experimental design, writing: review \& editing. All authors approved submission.

\section{Declaration of Interests}

The authors declare no competing interests.

\section{Acknowledgements}

We thank our collaborators of the KU Leuven killifish consortium Prof. Dr. L. Brendonck and Dr. T. Pinceel for providing African turquoise killifish eggs of the short-lived GRZ-AD strain. We thank Rony Van Aerschot, Arnold Van Den Eynde, and the KU Leuven killifish team for taking care of the fish facility. We also thank all our lab members (Dr. S. Gilissen, Dr. M. Hennes and L. Durieux) and the Seuntjens lab for timely discussions. We are grateful to the Genomics Core Leuven for both vital sequencing experiments and for assistance with data analysis.

\section{Funding}

This work was supported by Fonds voor Wetenschappelijk Onderzoek (FWO Vlaanderen; research grant number G0C2618N and personal fellowship to JVH (1S00318N), and an equipment grant from KU Leuven (KA/16/021).

\section{Figure Legends}

Figure 1. Cell type identification in 6 week-old adult killifish telencephalon.

(A) Seurat-based single-cell analysis strategy. (B) Pie chart depicting the diverse cell types and their relative cell numbers. (C1) TSNE plot at resolution 0.4 revealing 17 cell types. (C2) Plot with annotation of major cell clusters. (D) Relevant gene markers that identify the 17 cell types. Size of dot indicates the percentage of cells expressing the gene (0-100\%), color indicates expression level.

Abbreviations: NGP: non-glial progenitor, RR: retinal-like radial glia; OPC/OD: oligodendrocyte progenitor cell/Oligodendrocyte; NA: not assigned.

\section{Figure 2. PC-based subclusters and their marker identification.}

(A) Expression plot of markers used to differentiate progenitors (PCs) from other clusters. The pink area lacks PC marker expression. Pink: other, Blue: PC. (B) Iterative clustering of PCs revealed 7 cell sub-types. (C) Dot plot showing marker expression in every PC sub-cluster. Size of dot indicates the percentage of cells expressing the gene (0-100\%), color indicates expression level. (D) Cellular composition chart of the 7 PC sub-types. (E) Ridge plot showing density of cells expressing critical markers in various RG clusters including presumptive astroglia markers. The higher the peak, the more 
cells display a particular expression level. Multiple peaks indicate differential gene expression within the same cluster. (F) Ridgeplot showing density gradient of cells that express important NGP markers, therefore proving their distinction from radial glia types. Note: Sole cell peaks at expression 0 are indicative of low or zero expression of the gene in all cells of that cluster.

Abbreviations: NGP: non-glial progenitor, Inter-NGP: Intermediate cell NGP, Inter-RG: Intermediate Radial glia, NE-RG3: Neuroepithelial Radial glia 3, Astro-RG1: Astroglia-like Radial glia 1, AstroRG2: Astroglia-like Radial glia 2, EPD-RG4: Ependymal Radial glia 4.

Figure 3. TSNE plots and spatial distribution of typical RG marker expression as determined via in situ hybridization or immunohistochemistry.

(A1) Gene/Feature plot showing expression of SLC1A2, a marker for Astro-RG1. (A2) SLC1A2 insitu hybridization showing expression mostly localized to cell bodies at the ventricular surface of the telencephalon. (B1) Gene/Feature plot showing expression of CX43, a marker for Astro-RG1/AstroRG2. (B2) CX43 in-situ hybridization showing distinct expression in cell bodies at the ventricular surface and in fiber-like structures reminescant of radial glial fibers in the telencephalon. (C1) Gene/Feature plot showing expression of WNT8B, a marker for the starter radial glia type, NE-RG3. (C2) WNT8B immunohistochemistry reveals the ligand in cell bodies at the ventricular surface, in fiberlike structures and in the parenchyma of the telencephalon (D1) Gene/Feature plot showing expression of APOA1, a marker for the proposed ependymoglia, EPD-RG4. (D2) APOA1 in-situ hybridization reveals expression mostly localized in the ependymal lining of the ventricle. NOTE: Arrowheads point towards cells positive for the marker.

Figure 4. Lineage inference analysis of PCs indicates interplay between RG subtypes and NGP via Inter-NGP.

(A) PC based lineage inference analysis revealed 2 possible trajectories illustrated in (A1) and (A2). (B1) Pseudotime-ordered heatmap shows transcriptional profiles of top 30 variably expressed genes across the first lineage trajectory (A1). (B2) Pseudotime-ordered heatmap shows transcriptional profiles of top 30 variably expressed genes across the 2nd lineage trajectory (A2).

Abbreviations: NGP: non-glial progenitor, Inter-NGP: Intermediate cell NGP, Inter-RG: Intermediate Radial glia, NE-RG3: Neuroepithelial Radial glia 3, Astro-RG1: Astroglia-like Radial glia 1, AstroRG2: Astroglia-like Radial glia 2, EPD-RG4: Ependymal Radial glia 4.

Figure 5. NC-based subclusters and their marker identification.

(A) Iterative clustering of Neuronal clusters (NCs) revealed 11 cell sub-clusters. (B) Cellular composition chart of neuronal sub-clusters (NCs). (C) Violin plot showing expression of markers crucial for neural identity and neuronal differentiation. (D) Violin plot showing expression of mature neuronal markers. (E) Pseudotime ordering of cells shows 3 possible lineages (Lineage 1, 2, 3) starting from NE-RG3 cell cluster.

Abbreviations: Trans-N: Transitioning neuron, Ex-mN: Excitatory mature neuron, Ih-mN: Inhibitory mature neuron, NBN: Newly born neurons, NGP: non-glial progenitor, Inter-NGP: Intermediate cell NGP, NE-RG3: Neuroepithelial Radial glia 3

\section{Supplementary figure legends}

Supplementary Figure 1. Quality metrics of initial clustering. (A) Scatter plot showing no. of genes/transcripts detected per cell. (B) Violin plots for the number of gene per cell (nGene), number of reads per cell (nUMI), mitochondrial genes percentages (percent.mt) and ribosomal gene percentages (percent.ribo) in nUMI per cell. (C) Scatter plot and correlation between nUMI and nGene. (D) Elbow plot showing most variable principal components among all cells. (E) Heatmap showing the clustering of the 17 original different cell types (Fig. 1) as per their transcriptional profiles. (F) Heatmap showing the top expressed genes (ordered by p-value) per cell cluster.

Supplementary figure 2. Categorization of cell types. Feature plots of marker genes that identified other cell types such as in (A) all neurons [SCNB, SYP], (B) oligodendrocyte 
progenitors/oligodendrocytes (OPC/OD) [OLIG1, OLIG2], (C) microglia [AIF1, LCP1] and (D) vascular cells [TPM1, KLF2].

Supplementary figure 3. (A) Violin plots showing expression levels of progenitor-associated genes SOX2, SOX3 and PCNA in PC sub-clusters. (B) Dot plot showing expression profiling of genes associated with neural commitment and proliferation and/or quiescence. (C) Differential expression analysis of NEUROG1, NOTCH1, Notch ligands and FGFR genes in PC sub-clusters. (D) Differential expression analysis of conserved transcription factors and epigenetic regulators related to neurogenesis in PC sub-clusters. (E) Differential expression of BMP signaling associated genes. NOTE: Size of dot indicates the percentage of cells expressing the gene (0-100\%), color indicates expression level.

Supplementary figure 4. Pseudotime-based expression heat map of top 50 variably expressed genes across the 3 lineages (Lineage 1, 2 and 3) discovered within NC-based sub-clusters in (A), (B) and (C).

Supplementary figure 5. Gene marker expression comparisons between astroglia of killifish (AstroRG1, Astro-RG2) and zebrafish progenitor cell types (PC_0, PC_1, PC_2, PC_4).

\section{References}

1. Zupanc, G. K. H. Neurogenesis and neuronal regeneration in the adult fish brain. J. Comp. Physiol. A, Neuroethol. sensory, neural, Behav. Physiol. 192, 649-670 (2006).

2. Chapouton, P., Jagasia, R. \& Bally-Cuif, L. Adult neurogenesis in non-mammalian vertebrates. Bioessays 29, 745-757 (2007).

3. Lim, D. A. \& Alvarez-Buylla, A. The Adult Ventricular-Subventricular Zone (V-SVZ) and Olfactory Bulb (OB) Neurogenesis. Cold Spring Harb. Perspect. Biol. 8, (2016).

4. Ayana, R., Singh, S. \& Pati, S. Decoding Crucial LncRNAs Implicated in Neurogenesis and Neurological Disorders. Stem Cells Dev. 26, 541-553 (2017).

5. Feliciano, D. M., Bordey, A. \& Bonfanti, L. Noncanonical Sites of Adult Neurogenesis in the Mammalian Brain. Cold Spring Harb. Perspect. Biol. 7, a018846 (2015).

6. Bao, H. \& Song, J. Treating Brain Disorders by Targeting Adult Neural Stem Cells. Trends Mol. Med. 24, 991-1006 (2018).

7. Matthews, B. R. Memory Dysfunction. Contin. Lifelong Learn. Neurol. 21, (2015).

8. Howe, K. et al. The zebrafish reference genome sequence and its relationship to the human genome. Nature 496, 498-503 (2013).

9. Van houcke, J. et al. Aging impairs the essential contributions of non-glial progenitors to neurorepair in the dorsal telencephalon of the Killifish N. furzeri. bioRxiv 1-33 (2021). doi:10.1101/2021.02.26.433041

10. Diotel, N., Lübke, L., Strähle, U. \& Rastegar, S. Common and Distinct Features of Adult Neurogenesis and Regeneration in the Telencephalon of Zebrafish and Mammals . Frontiers in Neuroscience 14, 957 (2020).

11. Adolf, B. et al. Conserved and acquired features of adult neurogenesis in the zebrafish telencephalon. Dev. Biol. 295, 278-293 (2006).

12. Schmidt, R., Strähle, U. \& Scholpp, S. Neurogenesis in zebrafish - from embryo to adult. Neural Dev. 8, 3 (2013).

13. Dirian, L. et al. Spatial regionalization and heterochrony in the formation of adult pallial neural stem cells. Dev. Cell 30, 123-136 (2014).

14. Than-Trong, E. \& Bally-Cuif, L. Radial glia and neural progenitors in the adult zebrafish central nervous system. Glia 63, 1406-1428 (2015).

15. Daynac, M. \& Petritsch, C. K. Regulation of Asymmetric Cell Division in Mammalian Neural Stem and Cancer Precursor Cells BT - Asymmetric Cell Division in Development, Differentiation and Cancer. in (eds. Tassan, J.-P. \& Kubiak, J. Z.) 375-399 (Springer International Publishing, 2017). doi:10.1007/978-3-319-53150-2_17

16. Coolen, M., Labusch, M., Mannioui, A. \& Bally-Cuif, L. Mosaic Heterochrony in Neural Progenitors Sustains Accelerated Brain Growth and Neurogenesis in the Juvenile Killifish 
N. furzeri. Curr. Biol. 30, 736-745.e4 (2020).

17. Kim, Y., Nam, H. G. \& Valenzano, D. R. The short-lived African turquoise killifish: an emerging experimental model for ageing. Dis. Model. \&amp;amp; Mech. 9, 115 LP - 129 (2016).

18. Yaksi, E. \& Sandi, C. What brains do we study and why do we study them? Eur. J. Neurosci. 52, 4602-4603 (2020).

19. van den Brink, S. C. et al. Single-cell sequencing reveals dissociation-induced gene expression in tissue subpopulations. Nat. Methods 14, 935-936 (2017).

20. Batiuk, M. Y. et al. Identification of region-specific astrocyte subtypes at single cell resolution. Nat. Commun. 11, 1220 (2020).

21. Cuevas-Diaz Duran, R., Wang, C.-Y., Zheng, H., Deneen, B. \& Wu, J. Q. Brain RegionSpecific Gene Signatures Revealed by Distinct Astrocyte Subpopulations Unveil Links to Glioma and Neurodegenerative Diseases. eNeuro 6, ENEURO.0288-18.2019 (2019).

22. Malatesta, P., Appolloni, I. \& Calzolari, F. Radial glia and neural stem cells. Cell Tissue Res. 331, 165-178 (2008).

23. Bradley, R. A. et al. Regionally specified human pluripotent stem cell-derived astrocytes exhibit different molecular signatures and functional properties. Development 146, dev170910 (2019).

24. Lange, C. et al. Single cell sequencing of radial glia progeny reveals the diversity of newborn neurons in the adult zebrafish brain. Development 147, dev185595 (2020).

25. Kimura, A., Matsuda, T., Sakai, A., Murao, N. \& Nakashima, K. HMGB2 expression is associated with transition from a quiescent to an activated state of adult neural stem cells. Dev. Dyn. 247, 229-238 (2018).

26. Abraham, A. B. et al. Aberrant Neural Stem Cell Proliferation and Increased Adult Neurogenesis in Mice Lacking Chromatin Protein HMGB2. PLoS One 8, e84838 (2014).

27. Kageyama, R., Ohtsuka, T. \& Kobayashi, T. The Hes gene family: repressors and oscillators that orchestrate embryogenesis. Development 134, 1243 LP - 1251 (2007).

28. Jurisch-Yaksi, N., Yaksi, E. \& Kizil, C. Radial glia in the zebrafish brain: Functional, structural, and physiological comparison with the mammalian glia. Glia 68, 2451-2470 (2020).

29. Rothenaigner, I. et al. Clonal analysis by distinct viral vectors identifies bona fide neural stem cells in the adult zebrafish telencephalon and characterizes their division properties and fate. Development 138, 1459 LP - 1469 (2011).

30. Kroehne, V., Freudenreich, D., Hans, S., Kaslin, J. \& Brand, M. Regeneration of the adult zebrafish brain from neurogenic radial glia-type progenitors. Development 138, $4831 \mathrm{LP}-$ 4841 (2011).

31. Baumgart, M. et al. RNA-seq of the aging brain in the short-lived fish N. furzeri - conserved pathways and novel genes associated with neurogenesis. Aging Cell 13, 965-974 (2014).

32. Naef, V. et al. The Stemness Gene Mex3A Is a Key Regulator of Neuroblast Proliferation During Neurogenesis. Front. cell Dev. Biol. 8, 549533 (2020).

33. Cosacak, M. I. et al. Single-Cell Transcriptomics Analyses of Neural Stem Cell Heterogeneity and Contextual Plasticity in a Zebrafish Brain Model of Amyloid Toxicity. Cell Rep. 27, 1307-1318.e3 (2019).

34. McIntosh, R., Norris, J., Clarke, J. D. \& Alexandre, P. Spatial distribution and characterization of non-apical progenitors in the zebrafish embryo central nervous system. Open Biol. 7, (2017).

35. Kishimoto, N., Shimizu, K. \& Sawamoto, K. Neuronal regeneration in a zebrafish model of adult brain injury. Dis. Model. Mech. 5, 200-209 (2012).

36. La Rosa, C., Ghibaudi, M. \& Bonfanti, L. Newly Generated and Non-Newly Generated "Immature" Neurons in the Mammalian Brain: A Possible Reservoir of Young Cells to Prevent Brain Aging and Disease? Journal of Clinical Medicine 8, (2019).

37. Chen, J., Poskanzer, K. E., Freeman, M. R. \& Monk, K. R. Live-imaging of astrocyte morphogenesis and function in zebrafish neural circuits. Nat. Neurosci. 23, 1297-1306 (2020).

38. Than-Trong, E. et al. Lineage hierarchies and stochasticity ensure the long-term maintenance of adult neural stem cells. Sci. Adv. 6, eaaz5424 (2020). 
39. Vaccarino, F. M. et al. Changes in cerebral cortex size are governed by fibroblast growth factor during embryogenesis. Nat. Neurosci. 2, 246-253 (1999).

40. Seuntjens, E. et al. Sip1 regulates sequential fate decisions by feedback signaling from postmitotic neurons to progenitors. Nat. Neurosci. 12, 1373-1380 (2009).

41. Cahoy, J. D. et al. A transcriptome database for astrocytes, neurons, and oligodendrocytes: a new resource for understanding brain development and function. J. Neurosci. 28, 264-278 (2008).

42. Pringle, N. P. et al. Fgfr3 expression by astrocytes and their precursors: evidence that astrocytes and oligodendrocytes originate in distinct neuroepithelial domains. Development 130, 93-102 (2003).

43. Zhang, Y. et al. An RNA-sequencing transcriptome and splicing database of glia, neurons, and vascular cells of the cerebral cortex. J. Neurosci. 34, 11929-11947 (2014).

44. Bansod, S., Kageyama, R. \& Ohtsuka, T. Hes5 regulates the transition timing of neurogenesis and gliogenesis in mammalian neocortical development. Development 144, 3156-3167 (2017).

45. Magrinelli, E., Wagener, R. J. \& Jabaudon, D. Simultaneous production of diverse neuronal subtypes during early corticogenesis. bioRxiv 369678 (2018). doi:10.1101/369678

46. Wang, B. et al. Unveiling the complexity of the maize transcriptome by single-molecule longread sequencing. Nat. Commun. 7, 11708 (2016).

47. Eid, J. et al. Real-Time DNA Sequencing from Single Polymerase Molecules. Science (80-. ). 323, 133-138 (2009).

48. Ayana, R. et al. Manuscript under preparation. (2021).

49. Stuart, T. et al. Comprehensive Integration of Single-Cell Data. Cell 177, 1888-1902.e21 (2019).

50. Kobak, D. \& Berens, P. The art of using t-SNE for single-cell transcriptomics. Nat. Commun. 10, 5416 (2019).

51. Street, K. et al. Slingshot: cell lineage and pseudotime inference for single-cell transcriptomics. BMC Genomics 19, 477 (2018). 
bioRxiv preprint doi: https://doi.org/10.1101/2021.07.04.450918; this version posted July 4, 2021. The copyright holder for this preprint (which was not certified by peer review) is the author/funder, who has granted bioRxiv a license to display the preprint in perpetuity. It is made available under aCC-BY-NC-ND 4.0 International license.

\section{Figure 1}

A

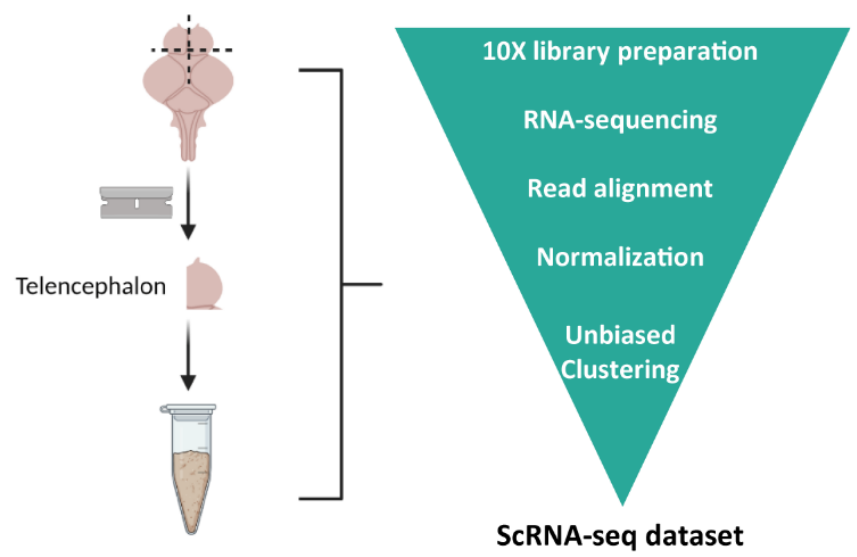

B

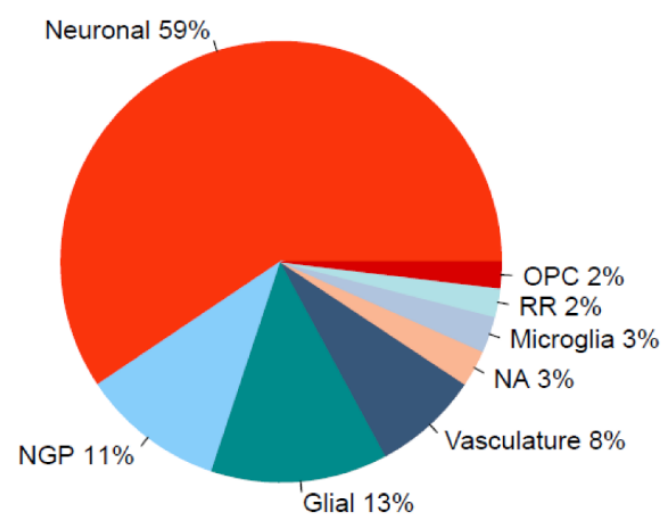

C1

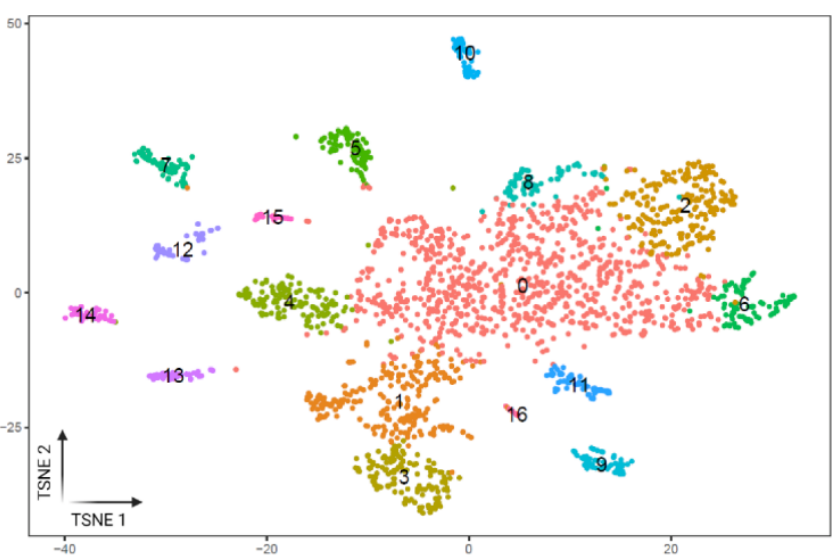

$\mathrm{C} 2$

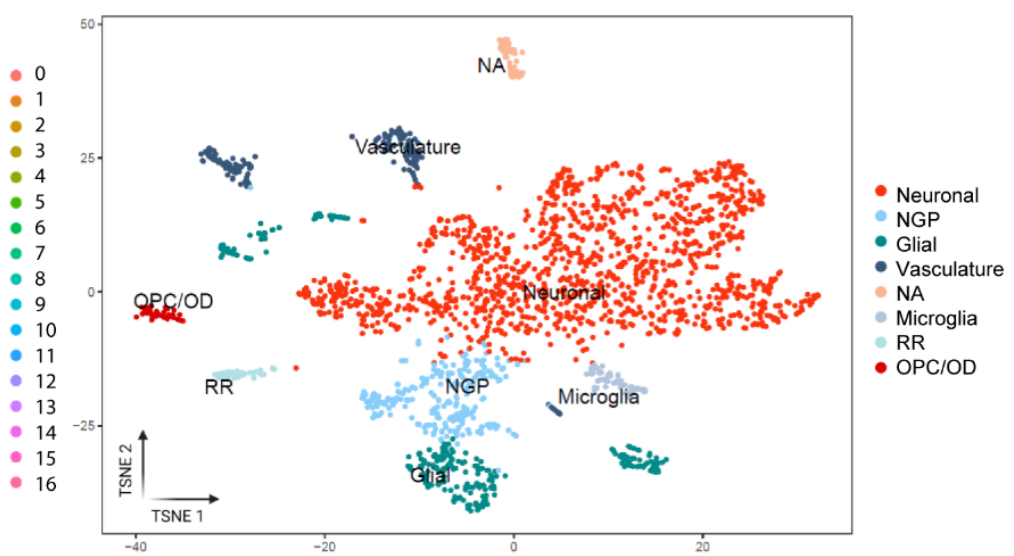

D

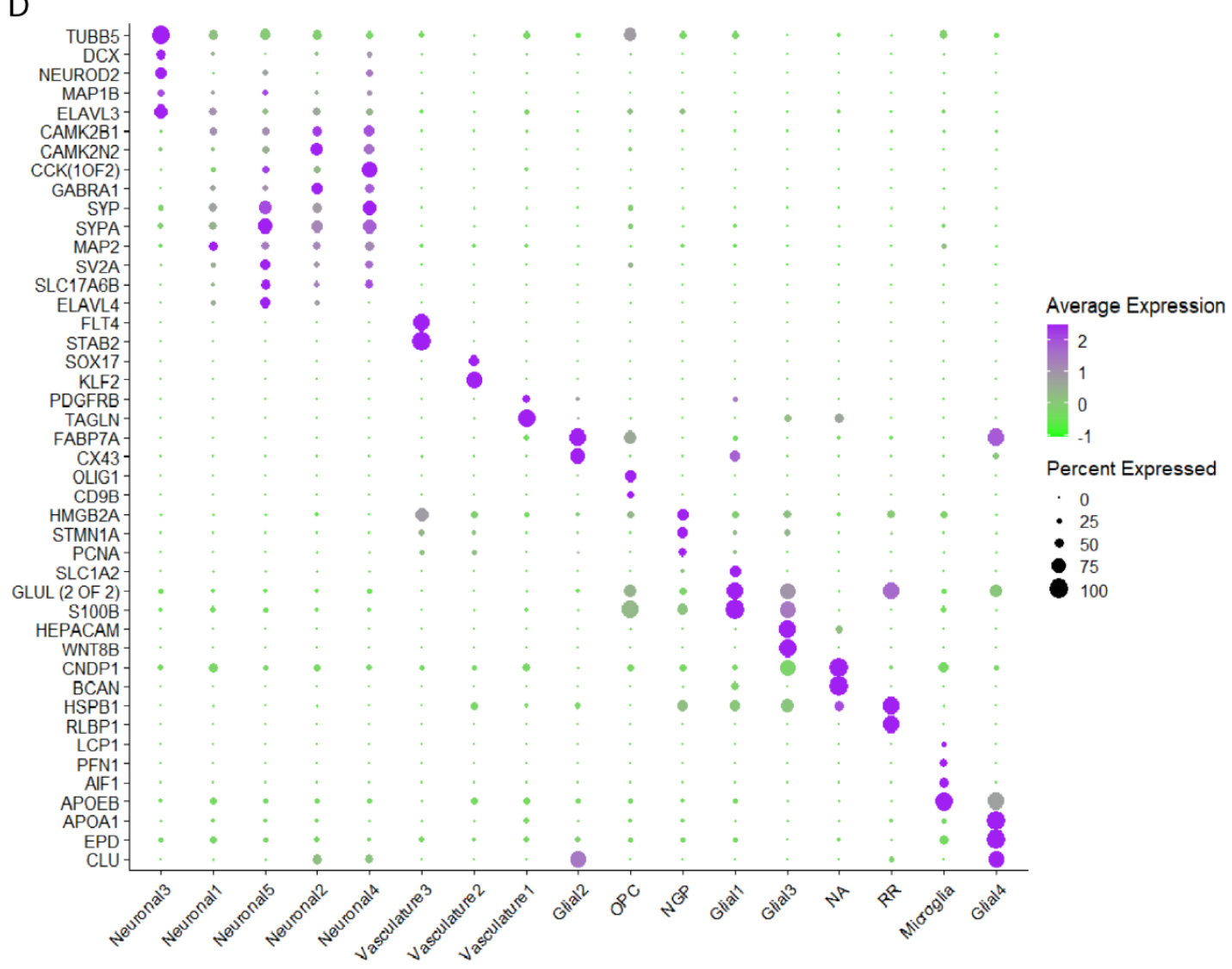


bioRxiv preprint doi: https://doi.org/10 1101/2021.07 04 450918; this version posted July 4 2021. The copyright holder for this preprint (which was not certified by peer review) is the author/funder, who has granted bioRxiv a license to display the preprint in perpetuity. It is made available under aCC-BY-NC-ND 4.0 International license.

Figure 2

A
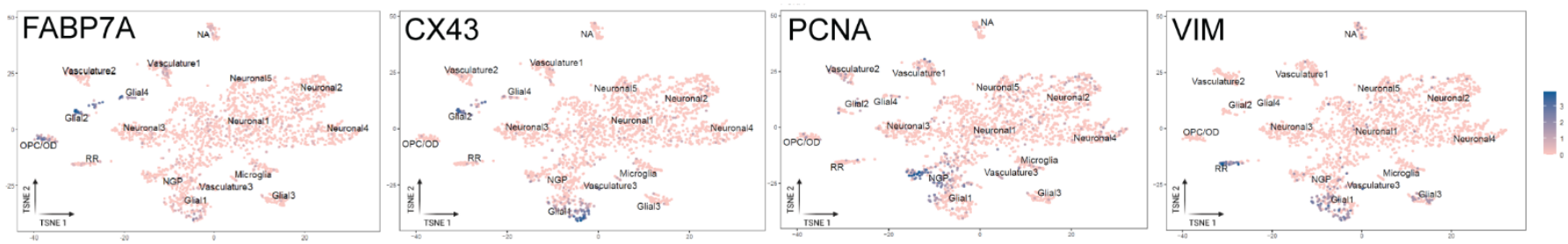

B

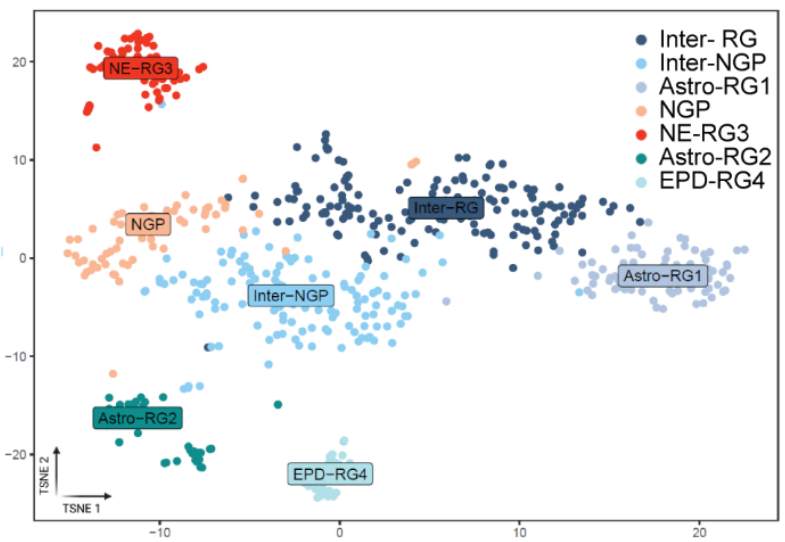

D

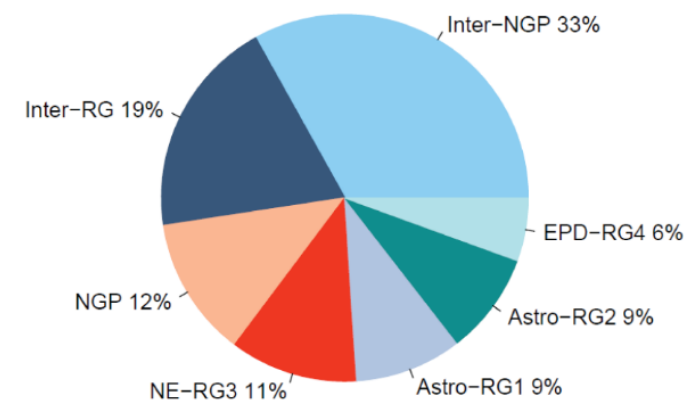

E
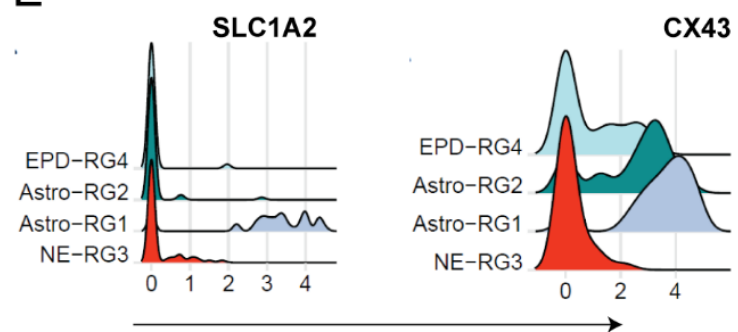

Expression level

F
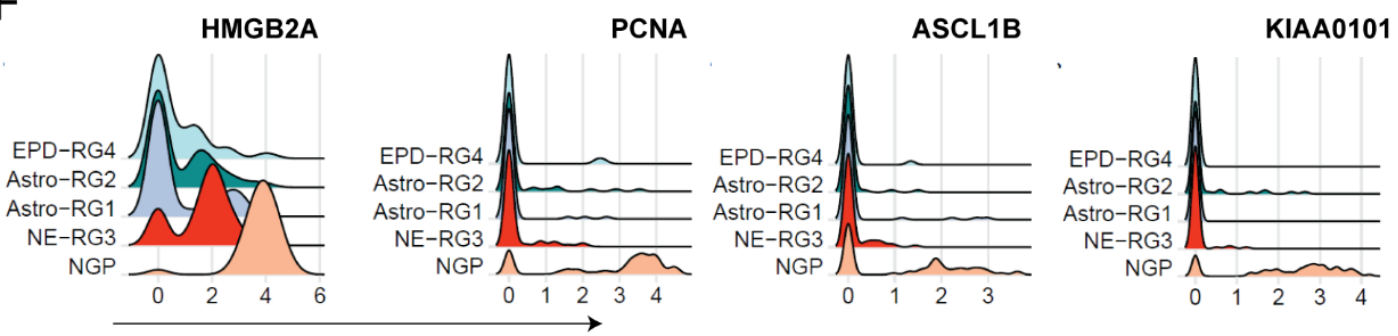

C

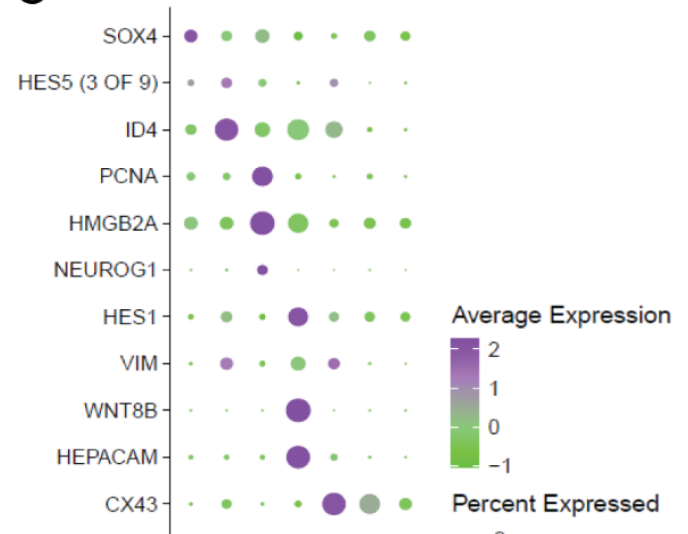

GLUL

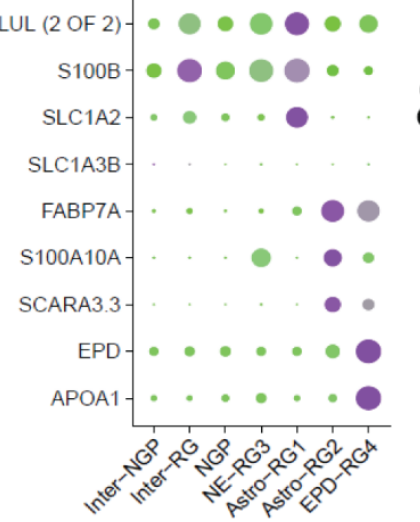

- 0
- 25

- 25

250
$-\quad 75$
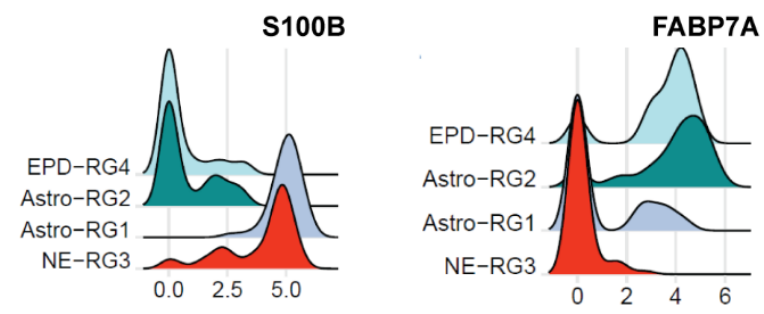

FABP7A

Expression level 
bioRxiv preprint doi: https://doi.org/10.1101/2021.07.04.450918; this version posted July 4, 2021. The copyright holder for this preprint (which was not certified by peer review) is the author/funder, who has granted bioRxiv a license to display the preprint in perpetuity. It is made available under aCC-BY-NC-ND 4.0 International license.

Figure 3

A1 Astro-RG1 - SLC1A2

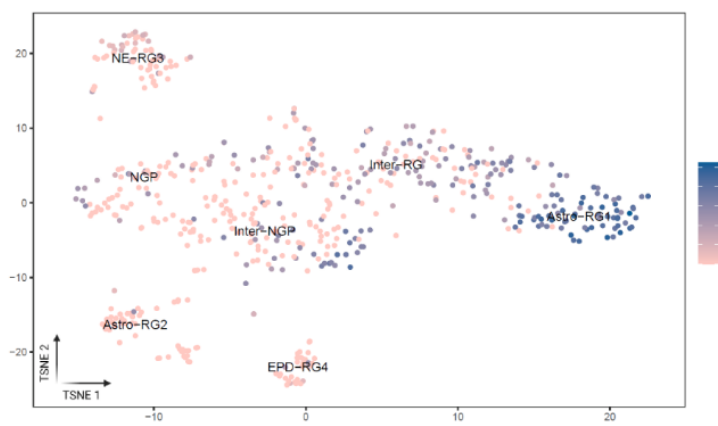

\section{B1 Astro-RG1/2 - CX43}

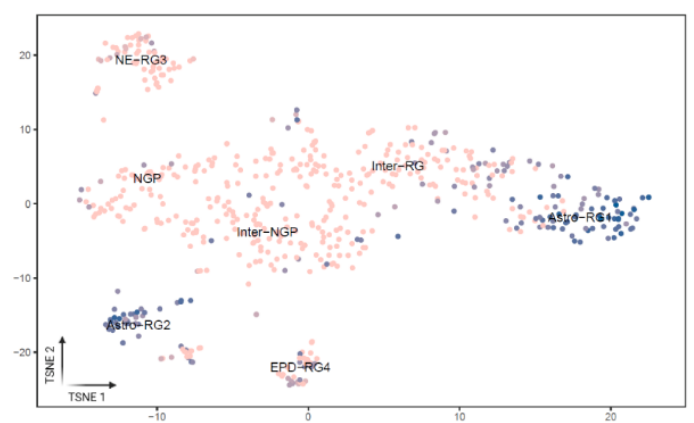

\section{C1 NE-RG3 - WNT8B}

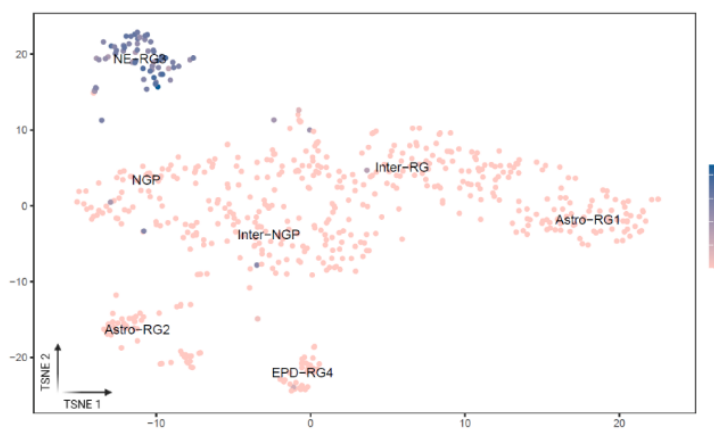

D1 EPD-RG4 - APOA1

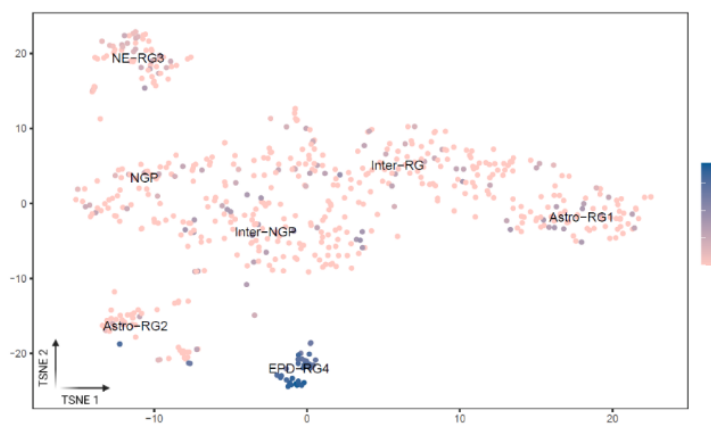

A2
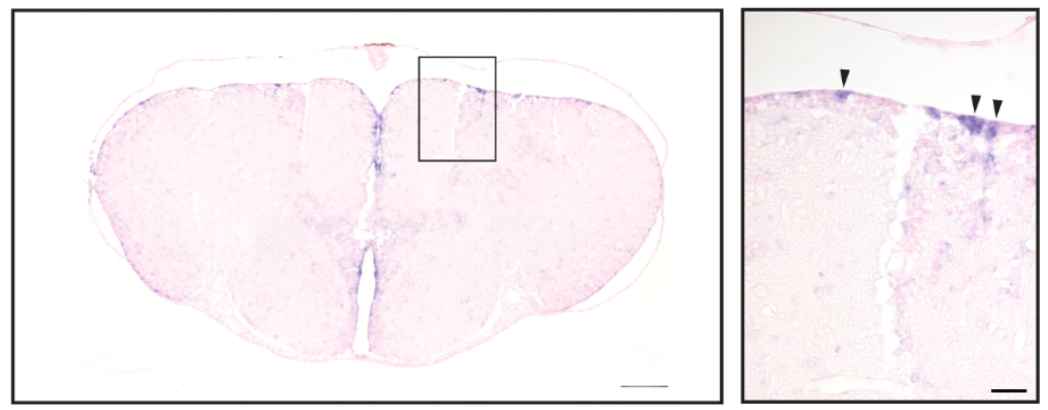

B2
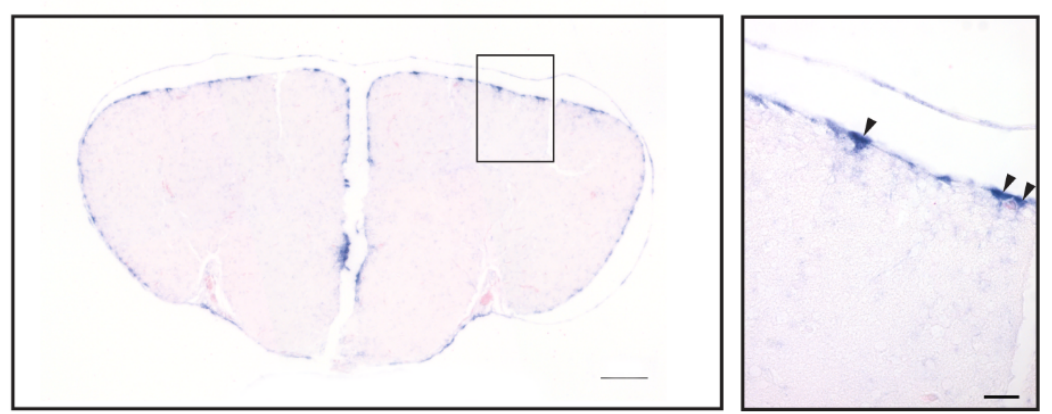

C2
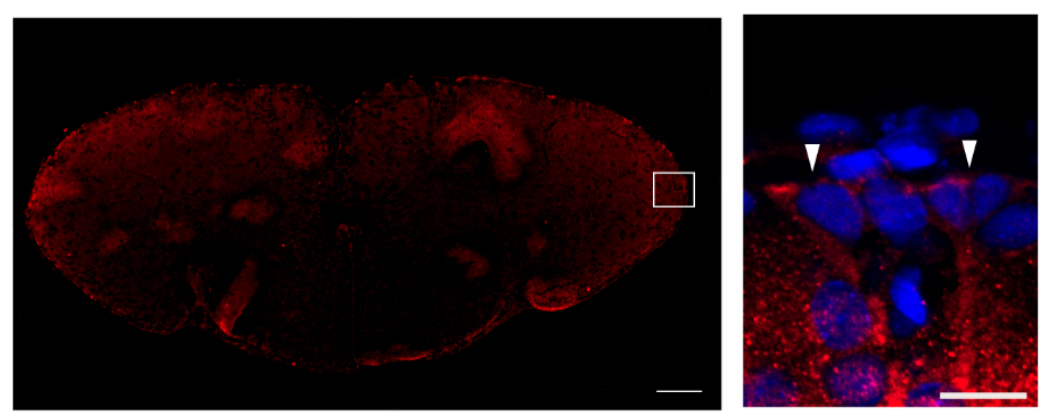

D2
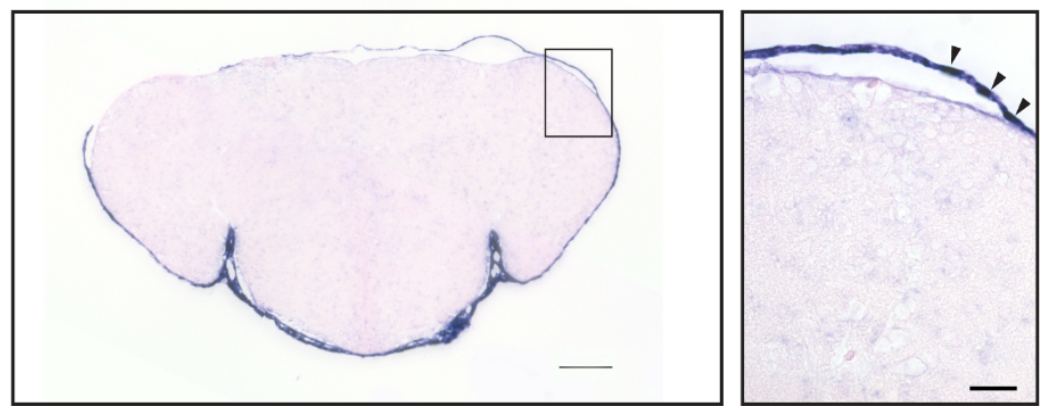
bioRxiv preprint doi: https://doi.org/10.1101/2021.07.04.450918; this version posted July 4, 2021. The copyright holder for this preprint (which was not certified by peer review) is the author/funder, who has granted bioRxiv a license to display the preprint in perpetuity. It is made available under aCC-BY-NC-ND 4.0 International license.

Figure 4

A1

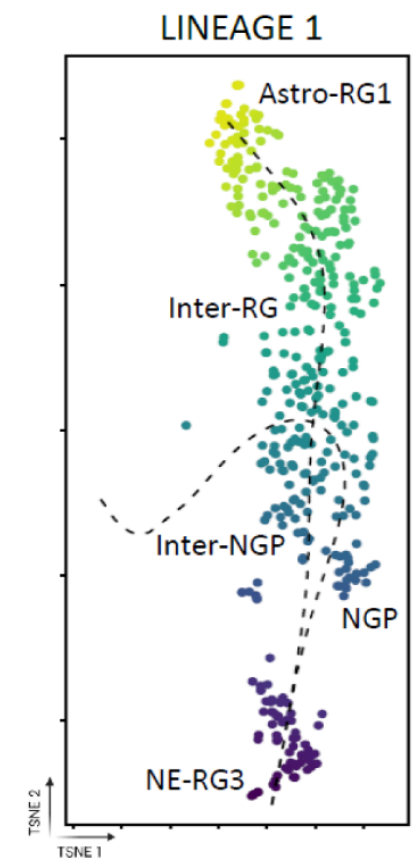

A2

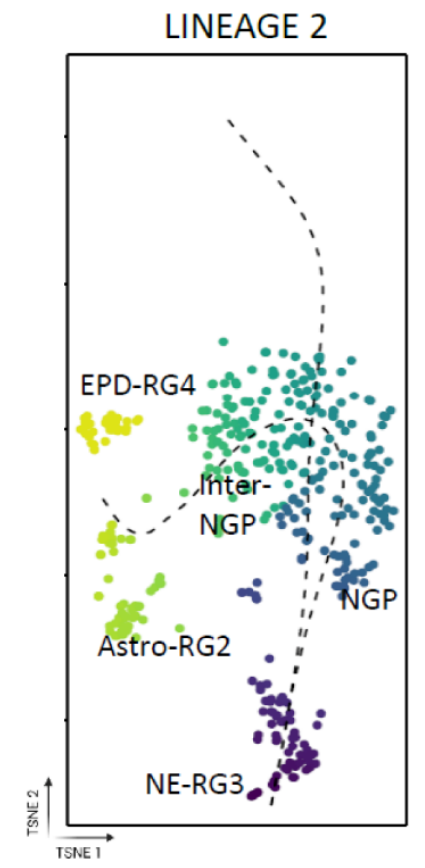

B2

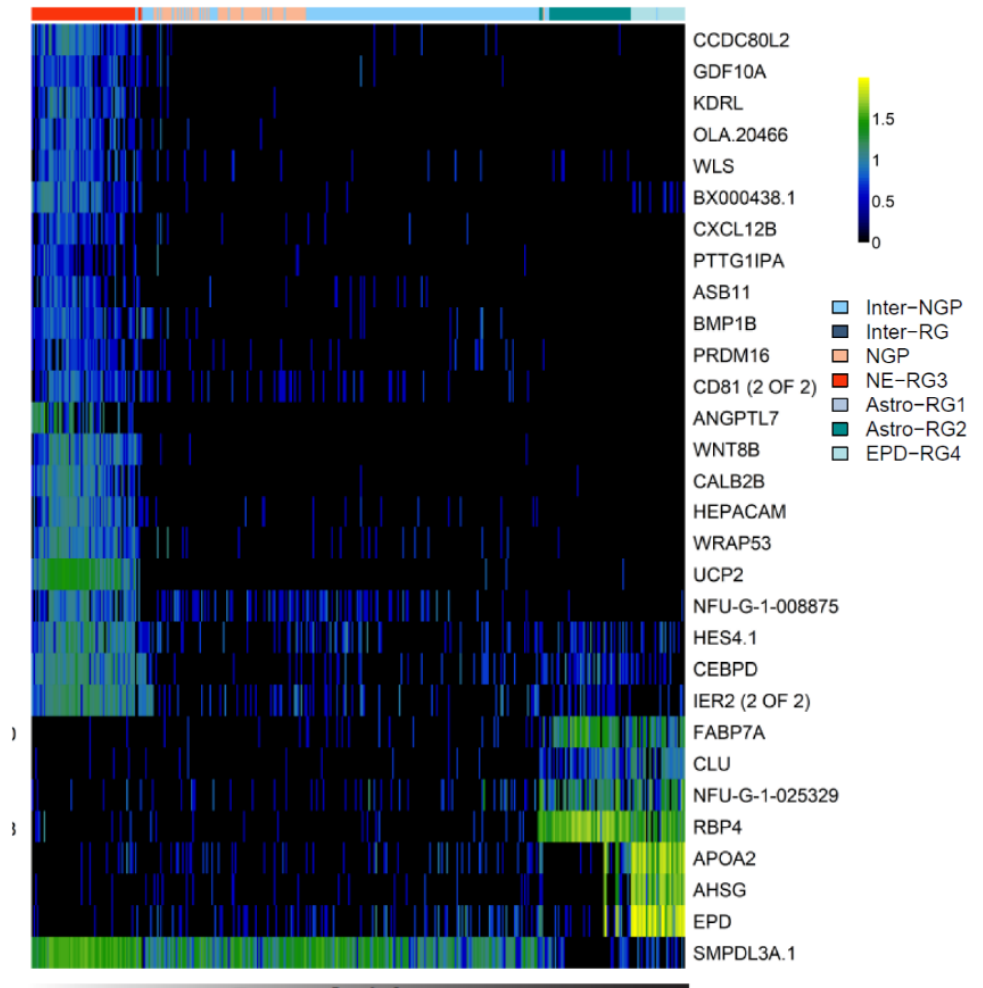

B1

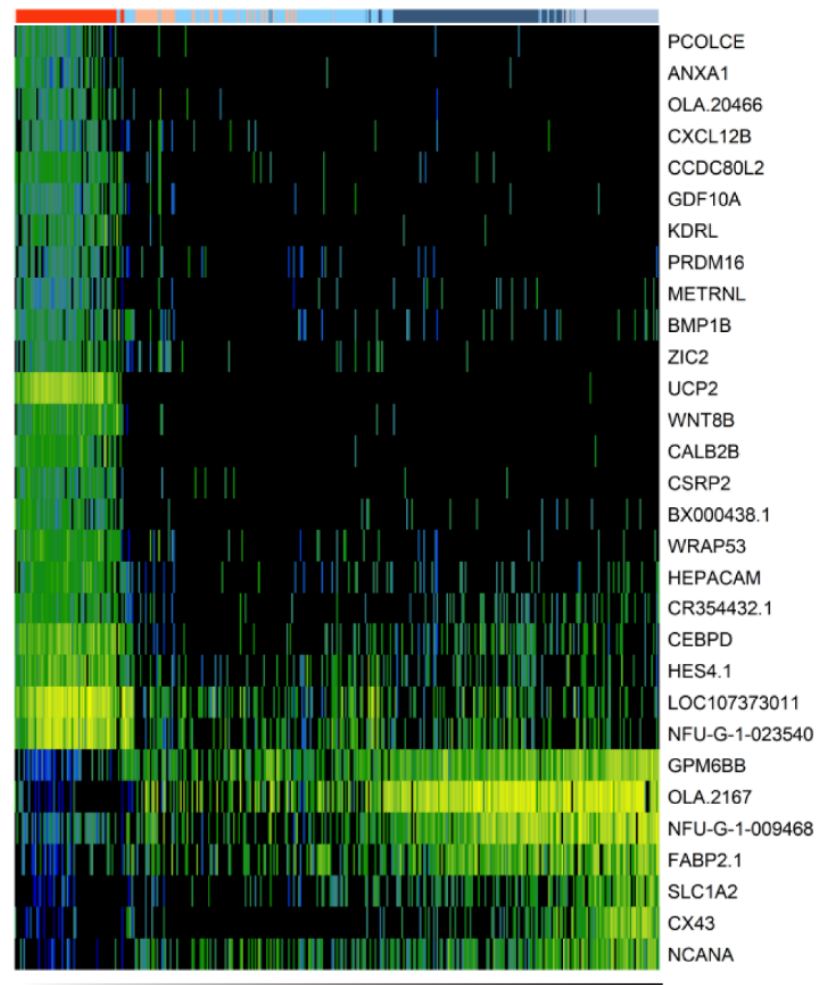

Pseudotime
Pseudotime 
bioRxiv preprint doi: https://doi.org/10.1101/2021.07.04 450918; this version posted July 4, 2021. The copyright holder for this preprint (which was not certified by peer review) is the author/funder, who has granted bioRxiv a license to display the preprint in perpetuity. It is made available under aCC-BY-NC-ND 4.0 International license.

Figure 5

A

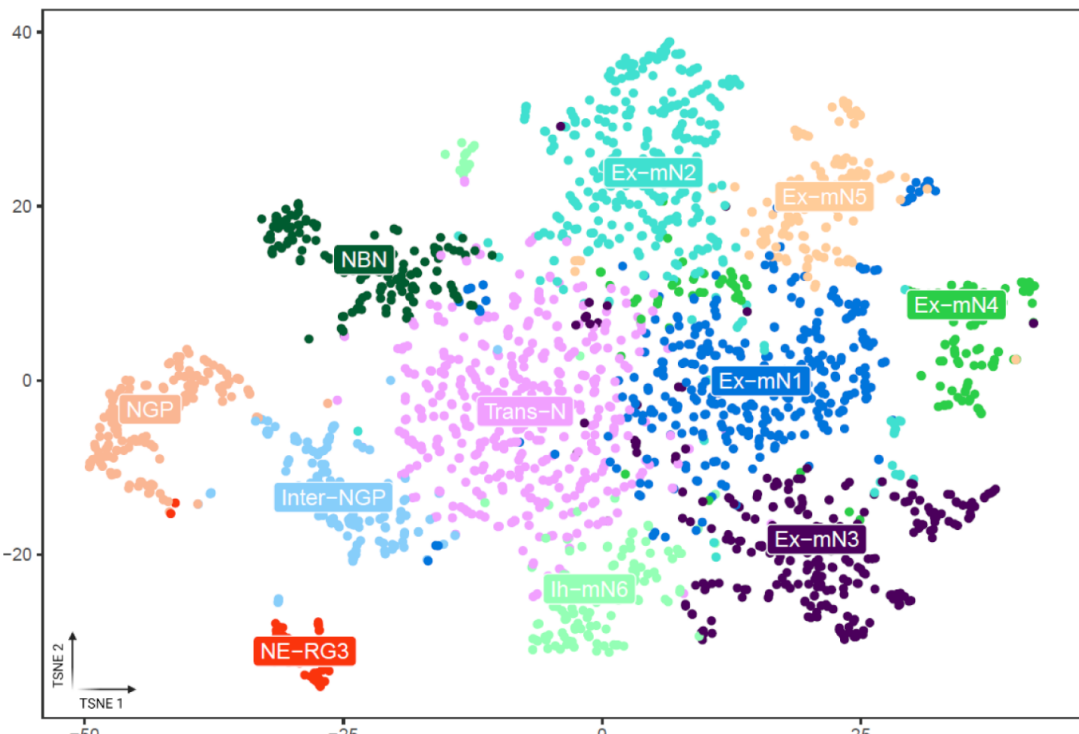

C

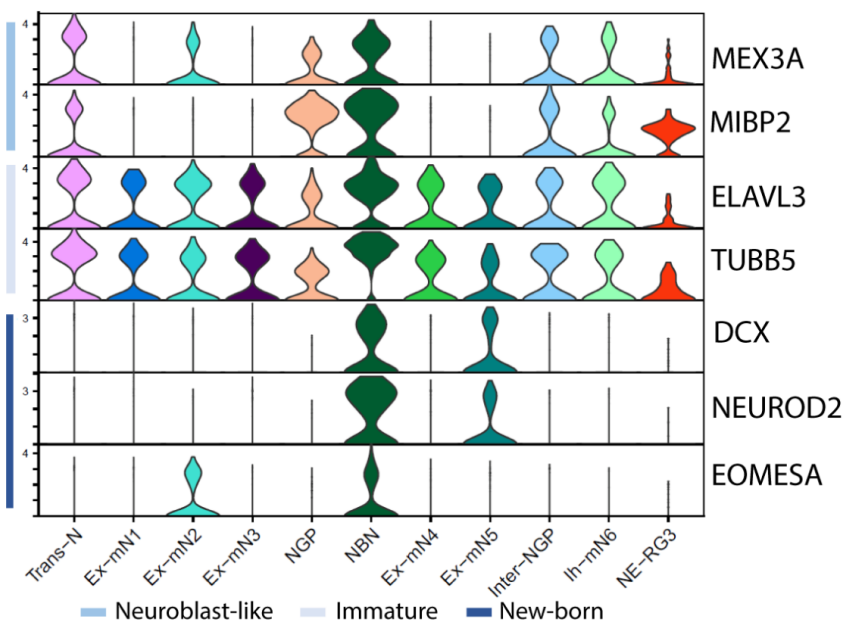

$\mathrm{E}$

LINEAGE 1

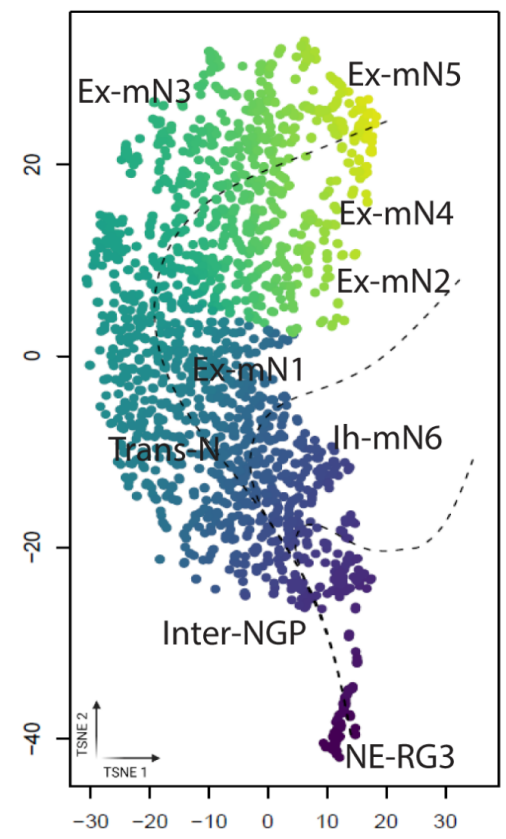

D

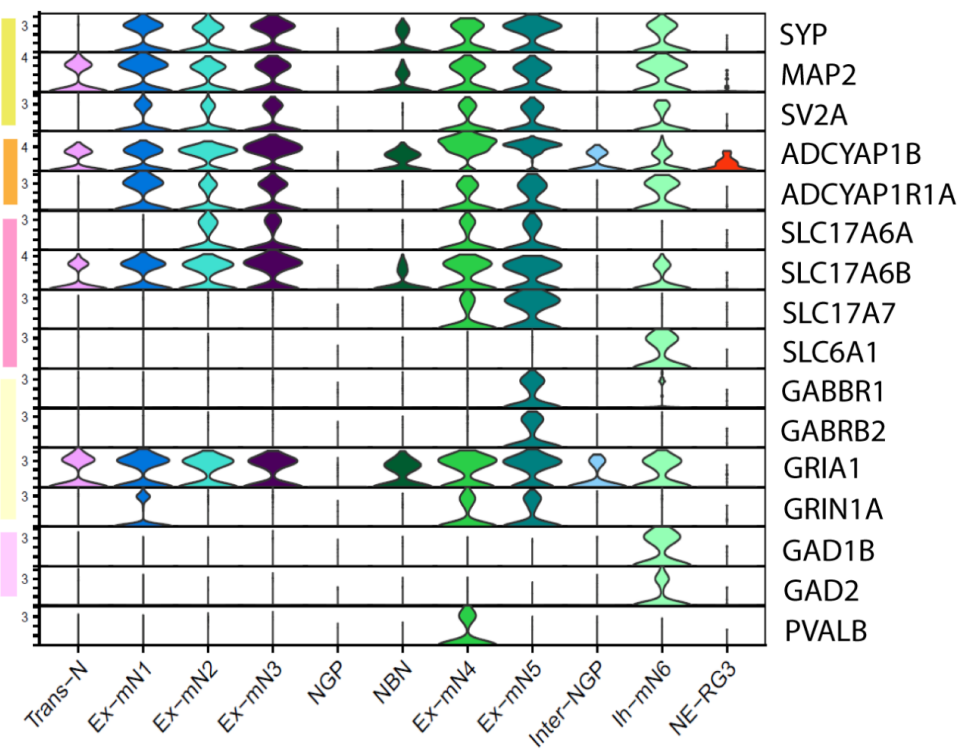

Pan-neuronal Neuropeptide/ Transporter Receptor Neurotransmitter Receptor

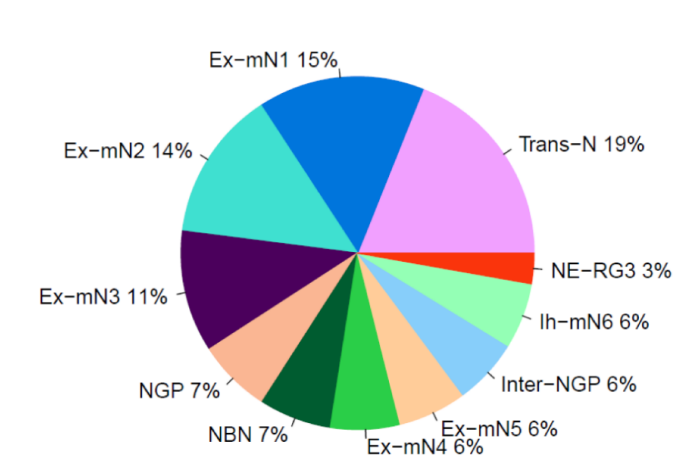

B

(1) 